A way forward in the study of the symmetry energy: experiment, theory, and observation

This content has been downloaded from IOPscience. Please scroll down to see the full text.

2014 J. Phys. G: Nucl. Part. Phys. 41093001

(http://iopscience.iop.org/0954-3899/41/9/093001)

View the table of contents for this issue, or go to the journal homepage for more

Download details:

IP Address: 129.57.75.213

This content was downloaded on 04/08/2014 at 20:10

Please note that terms and conditions apply. 
Topical Review

\title{
A way forward in the study of the symmetry energy: experiment, theory, and observation
}

\author{
C J Horowitz ${ }^{1}$, E F Brown ${ }^{2,8}$, Y Kim ${ }^{3}$, W G Lynch ${ }^{2}$, \\ R Michaels ${ }^{4}$, A Ono ${ }^{5}$, J Piekarewicz ${ }^{6}$, M B Tsang ${ }^{2}$ \\ and $\mathrm{H} \mathrm{H}$ Wolter $^{7}$
}

${ }^{1}$ Department of Physics and Nuclear Theory Center, Indiana University, Bloomington, IN 47405, USA

2 Department of Physics and Astronomy, and National Superconducting Cyclotron Laboratory, Michigan State University, East Lansing, MI 48824, USA

${ }^{3}$ Rare Isotope Science Project, Institute for Basic Science, Daejeon 305-811, Republic of Korea

${ }^{4}$ Thomas Jefferson National Accelerator Facility, Newport News, VA, USA

${ }^{5}$ Department of Physics, Tohoku University, Sendai 980-8578, Japan

${ }^{6}$ Department of Physics, Florida State University, Tallahassee, FL 32306, USA

${ }^{7}$ Fakultät für Physik, Universität München, Am Coulombwall 1, D-85748 Garching, Germany

E-mail: horowit@indiana.edu

Received 23 January 2014, revised 30 March 2014

Accepted for publication 28 April 2014

Published 22 July 2014

\begin{abstract}
The symmetry energy describes how the energy of nuclear matter rises as one goes away from equal numbers of neutrons and protons. This is very important to describe neutron rich matter in astrophysics. This article reviews our knowledge of the symmetry energy from theoretical calculations, nuclear structure measurements, heavy-ion collisions, and astronomical observations. We then present a roadmap to make progress in areas of relevance to the symmetry energy that promotes collaboration between the astrophysics and the nuclear physics communities.
\end{abstract}

Keywords: symmetry energy, neutron stars, heavy-ion collisions

(Some figures may appear in colour only in the online journal)

8 Joint Institute for Nuclear Astrophysics. 


\section{Introduction}

The liquid-drop formula of Bethe and Weizsäcker [1, 2] models the nucleus as an incompressible quantum drop consisting of $Z$ protons, $N$ neutrons, and mass number $A=Z+N$. In particular, the nuclear binding energy is expressed in terms of a handful of empirical parameters that capture the physics of a quantum drop. That is,

$$
B(Z, N)=a_{V} A-a_{S} A^{2 / 3}-a_{C} \frac{Z^{2}}{A^{1 / 3}}-a_{A} \frac{(N-Z)^{2}}{A}+\cdots
$$

The volume term $a_{V}$ represents the binding energy per nucleon of a large symmetric drop in the absence of long-range Coulomb forces. In turn, the next three terms denote bindingenergy corrections resulting from the development of a nuclear surface, the Coulomb repulsion among protons, and the Pauli exclusion principle and strong interactions that favor symmetric $(N=Z)$ systems. Although refinements to the mass formula have been made to account for the emergence of nuclear shells, the structure of this 75 year-old formula has remained practically unchanged.

In the thermodynamic limit in which both the number of nucleons and the volume are taken to infinity but their ratio remains fixed at the saturation density, the binding energy per nucleon may be written as

$$
\epsilon(\alpha) \equiv-\frac{B(Z, N)}{A}=-a_{V}+J \alpha^{2},
$$

where $J \equiv a_{A}$ and $\alpha=(N-Z) / A$ is the neutron-proton asymmetry. Note that we have neglected long-range Coulomb forces (which would render the drop unstable) and have assumed that both $Z$ and $N$ are individually conserved. Such a simple expression suggests that the binding energy per nucleon of a large symmetric drop of density $\rho_{0} \approx 0.15 \mathrm{fm}^{-3}$ is $a_{V} \approx 16 \mathrm{MeV}$ and that there is an energy cost of $J \approx 32 \mathrm{MeV}$ in converting all protons into neutrons. However, in reality the liquid drop is not incompressible, so the semi-empirical mass formula, while highly insightful, fails to describe the response of the liquid drop to density fluctuations. This information is contained in the equation of state (EOS) which dictates the dependence of the energy per nucleon on both the density and the neutron-proton asymmetry ${ }^{9}$. Following equation (2), we may write the EOS of asymmetric matter as

$$
\mathcal{E}(\rho, \alpha)=\mathcal{E}(\rho, \alpha=0)+S(\rho) \alpha^{2}+\cdots
$$

where $\mathcal{E}(\rho, \alpha=0)$ is the EOS of symmetric nuclear matter and $S(\rho)$ is the symmetry energy:

$$
S(\rho) \equiv \frac{1}{2}\left(\frac{\partial^{2} \mathcal{E}(\rho, \alpha)}{\partial \alpha^{2}}\right)_{\alpha=0} \approx \mathcal{E}(\rho, \alpha=1)-\mathcal{E}(\rho, \alpha=0) .
$$

Although the value of the symmetry energy at a density of $\rho \approx 0.1 \mathrm{fm}^{-3}$ is fairly well constrained by the masses of heavy nuclei, at present its density dependence is poorly known. Note that the EOS of asymmetric matter is mainly characterized by the density dependence of the symmetry energy $(\partial S / \partial \rho)$ which is essential for the understanding of the structure of neutron-rich nuclei, particle yields in heavy-ion collisions, and properties of neutron stars. To

9 The EOS and therefore the symmetry energy, are defined without model-dependent concepts. They include the effects of all kind of correlations in nuclear matter, such as the cluster correlations that are important at low densities. 
characterize the departure of the symmetry energy from its value at saturation, it is customary to perform a Taylor series expansion around saturation density. That is [3],

$$
S(\rho)=J+L x+\frac{1}{2} K_{\text {sym }} x^{2}+\cdots
$$

where $x=\left(\rho-\rho_{0}\right) / 3 \rho_{0}$. In particular, an enormous effort has been, and continues to be, devoted to determine the slope of the symmetry energy $L$,

$$
\left.L \equiv 3 \rho_{0}\left(\frac{\partial S}{\partial \rho}\right)\right|_{\rho_{0}} .
$$

The nuclear symmetry energy describes the increase in energy as matter changes away from a symmetric configuration of equal numbers of neutrons and protons (or up and down quarks). Observables involving moderately neutron-rich nuclei up to very neutron-rich astrophysical systems are highly sensitive to the density dependence of the symmetry energy. Interpretation of these observables is often hindered, however, by uncontrolled extrapolations. The following two examples serve to illustrate this point. The first example involves the composition of the outer crust of a neutron star which is known to emerge from a simple dynamics that is solely sensitive to the masses of moderate to extreme neutron-rich nuclei [4-6]. Whereas the masses of many of these nuclei have been measured accurately, the masses of highly exotic nuclei such as ${ }^{118} \mathrm{Kr}$ rely on extrapolations into regions far away from the range of validity of nuclearstructure models. Another example that will be discussed in greater detail in section 5 involves the simultaneous determination of the mass and radius of neutron stars from observations of $\mathrm{x}$-ray bursts. Although promising, at the present time systematic uncertainties in the analysis of x-ray bursters continue to hinder the reliable extraction of stellar radii. Thus the need for a systematic and comprehensive program to determine the density dependence of the symmetry energy becomes critical. Some important first steps along this direction have already been given; (see [7,8] and references contained therein). The aim of this contribution is to offer a way forward by fostering dialogue among the nuclear and astrophysical communities on how best to achieve this common goal.

Ideally, one would like to calculate the density dependence of the symmetry energy starting from quantum chromodynamics (QCD). Indeed, dense QCD is intimately connected to nuclear physics and astrophysics and offers a fertile testing ground for our understanding of quantum-field theories in the non-perturbative regime. Moreover, the rich phase diagram of baryonic matter is believed to exhibit unique and novel states of matter that should be directly predicted by QCD. Unfortunately, at present no theoretical framework is available to study dense matter from first principles. Whereas lattice QCD has been very successful in simulating the thermal component of the EOS, progress in describing matter at finite baryon density has been exceedingly slow. Thus, in practice one must rely on phenomenological approaches calibrated from both laboratory experiments and astrophysical observations. As such, existing and forthcoming rare-isotope facilities will play an essential role in elucidating the nature of the phase diagram of strongly interacting matter and will provide critical inputs for refining the theoretical models of dense matter.

The density dependence of the symmetry energy plays a critical role in shaping the structure of finite nuclei. In particular, the neutron-skin thickness of heavy nuclei is highly sensitive to the difference between the symmetry energy at saturation density (as in the nuclear core) and the symmetry energy at lower densities (as in the nuclear surface). Ultimately, the thickness of the neutron skin emerges from a competition between the surface tension and the slope of the symmetry energy $L$. Moreover, the electric dipole polarizability, described below, is also highly sensitive to the density dependence of the symmetry energy. The dipole polarizability is an ideal complement to the neutron-skin thickness because the symmetry energy acts as the restoring force. 
The density dependence of the symmetry energy also has a profound impact on a variety of astrophysical phenomena. At very low densities, uniform neutron-rich matter becomes unstable against cluster formation. The onset of the instability and the formation of clusters is controlled by the symmetry energy; this may be important during the collapse of the core of a massive star or during the merger of two neutron stars. As such, the symmetry energy may play an important role in setting the conditions under which r-process nucleosynthesis occurs. Moreover, for densities of $\rho \approx(2-3) \rho_{0}$ a significant component of the pressure of neutron-rich matter is determined by $\partial S / \partial \rho$, and this in turn determines the neutron-star radius. Finally, at even higher densities, the symmetry energy controls the proton fraction, which in turn dictates whether enhanced neutrino cooling of neutron stars, via the direct Urca process is possible [9], see section 5.2.

In the laboratory, the density dependence of the symmetry energy can be probed via heavy-ion collisions at different beam energies and with nuclei having a wide range of neutronproton asymmetries. Indeed, heavy-ion collisions provide the only means to study the EOS of asymmetric matter under controlled laboratory conditions. In particular, low-energy heavy-ion collisions can produce warm, dilute neutron-rich matter that closely resemble the conditions in the neutrinosphere (i.e., the surface of last neutrino scattering) in a core-collapse supernova. At higher beam energies, collisions of neutron-rich heavy ions may shed light on the symmetry energy at densities of $\rho \approx 2 \rho_{0}$, a region that is critical in the development of the neutron-star radius. At these energies, pion production and particle flow may serve as useful probes of the symmetry energy at high densities. Critical to the success of this endeavor, however, is the reliability of transport models in distilling details of the symmetry energy from heavy-ion observables.

The purpose of this review is to provide a roadmap for future progress in studying the symmetry energy. After the brief motivation presented in this Introduction, we continue by discussing recent progress and ongoing efforts in a variety of topics of relevance to the symmetry energy, such as microscopic calculations, dilute neutron-rich matter, nuclear structure, neutron-star matter, and heavy-ion collisions. We then conclude with a description of our vision for a 'way forward' in each of these areas.

\section{Calculations of the symmetry energy}

Our inability to calculate the symmetry energy from first principles increases the importance of both laboratory experiments and astronomical observations. One can, however, calculate $S$ from first principles in the high temperature, low density regime. Furthermore, chiral effective field theory provides promising results for $S$ at $\rho \lesssim \rho_{0}$. At higher densities, a variety of calculations exist but these rely on mostly phenomenological approaches. In what follows, we review calculations of the symmetry energy at high temperatures in section 2.1 , at densities near $\rho_{0}$ in section 2.2, and finally at high densities in section 2.3.

\subsection{Symmetry energy at high temperatures and low densities}

At temperatures $T \gtrsim \mathrm{MeV}$ and densities $\rho \ll \rho_{0}$, one can calculate $S$ exactly using a virial expansion [10]. In this limit the EOS may be expanded in powers of the fugacity $z_{i}=\exp \left(\mu_{i} / T\right)$, where $\mu_{i}$ is the chemical potential for species $i$. The virial expansion is valid near the classical limit of $z_{i} \ll 1$. Quadratic coefficients of the virial expansion are calculated from nucleon-nucleon (NN), nucleon-light-cluster, and light-cluster-light-cluster elastic scattering phase shifts. Note that there are important contributions from alpha particles that can significantly increase the symmetry energy. The symmetry energy computed from the 
virial expansion provides an exact critical benchmark for supernova simulations (see section 3) that can be probed in the laboratory with heavy-ion collisions; (see, for example, [11]). At temperatures $\gtrsim 50 \mathrm{MeV}$ or above, one can extend the virial expansion to also include pion degrees of freedom. At low densities and even higher temperatures $T \gtrsim 100 \mathrm{MeV}$, where $\mu_{i} / T \ll 1$, one can calculate $S$ directly from QCD simulations. In general, lattice QCD can calculate the EOS for any temperature-in the limit of zero baryon density. Although lattice simulations at arbitrary densities are hindered by the conspicuous 'sign problem', progress in this area has been achieved by expanding around the zero baryon density limit. Indeed, if the EOS is expanded in powers of the chemical potential to the temperature, lattice QCD simulations have been successful in calculating the relevant coefficients through order $\left(\mu_{i} / T\right)^{4}$ $[12,13]$. In a future work, we will use these coefficients to constrain the symmetry energy at high temperatures. Although symmetry energy effects at high temperatures may be small relative to the large thermal energies, these model-independent QCD results provide important benchmarks in the calibration of theoretical models.

\subsection{Symmetry energy near saturation density}

Chiral effective field theory is a powerful approach in which nuclear interactions are systematically expanded in powers of the momentum transfer over a typical chiral (e.g., pion mass) scale. Perhaps the most important feature of the chiral approach is its hierarchical nature. That is, provided that the chiral expansion converges, two-nucleon forces dominate over three-nucleon forces, which in turn are more important than four-nucleon forces, and so on. This allows one to perform many-body calculations for which the complexity of the Hamiltonian is manageable. This approach has been used successfully for nuclear structure, see for example [14]. Recently, [15] used many-body perturbation theory to calculate the energy of infinite neutron matter to order N3LO by including two-, three-, and four-nucleon forces. Moreover, given the systematic nature of the expansion, estimates of the theoretical uncertainties were also provided. To provide estimates for both the symmetry energy $J$ and slope $L$ at saturation density, they approximate the symmetry energy (as in equation (4)) as the difference between the energy of pure neutron matter-which they calculate-and the energy of symmetric nuclear matter, which they do not calculate but instead adopt the empirical saturation point. The following values were reported [15]: $S_{0} \equiv J=(28.9-34.9) \mathrm{MeV}$ and $L=(43-67) \mathrm{MeV}$. However, note that do not calculate the EOS of pure neutron matter much beyond saturation density, as the chiral expansion may converge poorly at higher densities.

\subsection{Symmetry energy at high densities}

At present neither laboratory experiments nor astronomical observations place stringent constraints on the symmetry energy at high densities. Calculations of $S$ at high densities are hindered by large uncertainties related to the poor convergence of the chiral expansion. For example, [16] calculate the energy of pure neutron matter up to densities of $\rho \gtrsim 3 \rho_{0}$ using quantum Monte Carlo techniques with phenomenological two- and three-nucleon forces. In particular, by varying the three-nucleon force, they find a sharp linear correlation between $S_{0}$ and $L$. However, without proper theoretical guidance it is unclear whether the form of their three-nucleon force is reliable. Moreover, without a proper expansion (chiral or otherwise) it is also unclear whether four-nucleon (or higher order) forces could make a significant contribution at high densities. Thus, it is likely that these calculations may have large theoretical uncertainties at high densities. Note that there are also calculations of the symmetry energy using Brueckner many-body theory with phenomenological two- and three-nucleon forces, 
see for example $[17,18]$. However, these calculations may also suffer from large uncertainties related to their phenomenological Hamiltonians and the role of many-nucleon forces.

At this time, we have no clear $a b$ initio method to calculate the symmetry energy at high densities. Perhaps very phenomenological approaches can still provide useful insight. As examples, we mention two very different approaches. In [19, 20] a lattice of Skyrmions is used to calculate the symmetry energy, while [21] calculates the symmetry energy in holographic QCD.

We now summarize section 2. Chiral effective field theory provides a promising way to calculate the symmetry energy for uniform matter at low densities. At very low densities, matter is likely nonuniform and this must be taken into account when calculating the symmetry energy. One way to do this is by including clusters in a virial expansion. Unfortunately, all present calculations of the symmetry energy at high densities (above nuclear saturation) may have large uncertainties related to the form of the interactions. Therefore one may need to rely instead on phenomenological models, astrophysical observations, and heavy ion experiments.

\section{Symmetry energy at very low densities in non-uniform matter}

At low densities, uniform nuclear matter becomes unstable against cluster formation. Indeed, at densities of $\rho \lesssim \rho_{0} / 2$ the inter-nucleon separation becomes comparable to the range of the NN interaction, so it becomes energetically favorable for the system to fragment into neutron-rich clusters. Cluster formation significantly increases the symmetry energy at very low densities and this may be of relevance to the modeling of core collapse supernovae (CCSN). These giant stellar explosions radiate away the large gravitational binding energy of a neutron star $\left(\sim 100 \mathrm{MeV}\right.$ nucleon $\left.{ }^{-1}\right)$ by emitting $\approx 10^{58}$ neutrinos. Much of the 'action' in CCSN happens near the neutrinosphere, which defines the transition region between interacting and free-streaming neutrinos. The neutrinosphere is composed of a warm low-density gas of neutron-rich matter at temperatures of $T \sim 5 \mathrm{MeV}$ and densities of $\rho \sim 0.01 \rho_{0}$. Here the neutrino mean-free path becomes comparable to the size of the system.

Recently, several groups have come to appreciate that in addition to a free gas of neutrons and protons, the neutrinosphere may also contain light nuclei [10, 22] and may display important many-body correlations [23]. These many-body effects can have a significant impact on neutrino transport by modifying the neutrino opacity and ultimately the emitted neutrino spectra [24, 25]. In particular, this may be important for nucleosynthesis in the innermost regions of CCSN.

Remarkably, many properties of the neutrinosphere can be directly reproduced in the laboratory with heavy-ion collisions. Temperatures of about $5 \mathrm{MeV}$ are easy to achieve, while low subnuclear densities can be studied, for example, by observing intermediate velocity fragments from peripheral collisions [11]. Perhaps the most difficult property to simulate in these collisions is the large neutron-to-proton ratio present in the neutrinosphere. However, new and forthcoming radioactive beam facilities, such as RIBF (Radioactive Isotope Beam Facility) (already in operation) and FRIB (Facility for Rare Isotope Beams) (planned for 2020), allow heavy-ion collisions involving systems with widely varying neutron-proton asymmetries. By comparing results between neutron-deficient and neutron-rich systems, one should be able to extrapolate to systems with extreme neutron excess; see section 6. By measuring the composition of light clusters, these laboratory experiments should be able to infer the symmetry energy and the corresponding EOS of asymmetric nuclear matter at very low density. In turn, these measurements should provide critical inputs for new microscopic approaches to warm, dilute matter that face the challenge of accounting for many-body correlations and for the concomitant development of nuclear clusters. 


\section{Symmetry energy and nuclear structure}

\subsection{Neutron skins}

Starting with the pioneering work of Hofstadter in the late 1950's [26] and continuing to this day, measurements of charge distributions of nuclei using elastic electron scattering have provided knowledge of charge radii with remarkable accuracy across the nuclear chart $[27,28]$. In contrast, probing neutron densities has traditionally relied on hadronic experiments that are hindered by large and uncontrolled uncertainties. Compared to electron scattering where the reaction mechanism (i.e., one-photon exchange) is well understood, the reaction mechanism in the case of hadronic probes is complex due to the non-perturbative nature of the scattering process [29, 30]. Moreover, whereas the underlying elementary electronnucleon interaction remains largely unchanged in the nuclear medium, the in-medium hadronnucleon interaction acquires non-trivial density and momentum dependence. Finally, although Coulomb distortions may be significant in the case of electron scattering from heavy nuclei, the Coulomb interaction, unlike the hadron-nucleus optical potential, is known exactly. These large hadronic uncertainties make impossible to cleanly separate the reaction dynamics from nuclear-structure effects. Indeed, the uncertainties are so dramatic, that earlier studies of elastic proton scattering from both ${ }^{40} \mathrm{Ca}$ and ${ }^{208} \mathrm{~Pb}$ at a variety of incident energies yield hugely contradictory results in the extraction of their respective neutron radii [29]. Also note that in kinematic regions where the uncertainties are minimized-but certainly not eliminated-proton-nucleus scattering appears insensitive to even gross details of the neutron density [31].

Recently, the Lead Radius Experiment (PREX) at the Thomas Jefferson National Accelerator Facility (Jefferson Lab) has pioneered parity-violating measurements of neutron radii by relying on the significantly larger weak charge of the neutron relative to that of the proton $[32,33]$. Measurements of the parity-violating asymmetry $A_{\mathrm{PV}}$ at Jefferson Lab using longitudinally polarized electrons is an established technique that has been used successfully to probe the quark structure of the nucleon [34-42]. The parity-violating asymmetry is defined as the difference in the cross section between right- and left-handed longitudinally polarized electrons relative to their sum. That is,

$$
A_{\mathrm{PV}}=\frac{\sigma_{R}-\sigma_{L}}{\sigma_{R}+\sigma_{L}}
$$

This powerful technique provides a unique opportunity to measure the weak charge form factor of the nucleus - and hence its neutron radius $R_{n}$-in a relatively clean and model-independent way [43-46]. Indeed, PREX has provided for the first time model-independent evidenceat the $1.8 \sigma$ level-in favor of a neutron-rich skin in ${ }^{208} \mathrm{~Pb}$ and successfully demonstrated the feasibility of this technique for measuring neutron densities with an excellent control of systematic errors $[32,33]$. The neutron-skin thickness, defined as the difference between the neutron $\left(R_{n}\right)$ and proton $\left(R_{p}\right)$ root-mean-square radii was reported to be

$$
R_{\mathrm{skin}}^{208}=R_{n}^{208}-R_{p}^{208}=0.33_{-0.18}^{+0.16} \mathrm{fm} .
$$

In a follow-up already approved experiment 'PREX-II', the uncertainty in the determination of $R_{n}^{208}$ will be reduced by a factor of 3 , to $\pm 0.06 \mathrm{fm}$.

The neutron-skin thickness of ${ }^{208} \mathrm{~Pb}$ is strongly sensitive to the density dependence of the symmetry energy. In particular, $R_{\text {skin }}^{208}$ is strongly correlated to the slope of the symmetry energy $L$ [47]. This correlation is strong because it emerges from simple, yet robust, physical arguments. In the spirit of the liquid-drop model, surface tension favors the formation of a 
spherical drop of uniform equilibrium density. However, for a neutron-rich system it is unclear whether the extra neutrons should reside in the surface or in the core. Placing them in the core is favored by surface tension but disfavored by the symmetry energy, which is large at saturation density. Conversely, moving them to the surface increases the surface tension but reduces the symmetry energy. Hence, the neutron-rich skin of a heavy nucleus emerges from a competition between the surface tension and the difference between the symmetry energy at saturation density and at a lower surface density. In particular, for a stiff symmetry energy, namely one that increases rapidly with density, it is energetically favorable to move most of the the neutrons to the surface where the symmetry energy is low; this generates a thick neutron skin.

Given that at zero temperature the difference between the symmetry energy at two neighboring density points is directly proportional to the symmetry pressure $L$, a strong correlation between $R_{\text {skin }}^{208}$ and $L$ is expected. Indeed, Roca-Maza et al [38] find a strong correlation (with a correlation coefficient of $r=0.979$ ) between $R_{\text {skin }}^{208}$ and $L$ using a large and representative set of energy density functionals (EDFs). A linear fit to the predictions of all the models displayed in [38] yields:

$$
R_{\text {skin }}^{208}=\frac{r_{\mathrm{s}}}{2}\left(\frac{L+L_{\mathrm{s}} \pm \delta L_{\mathrm{s}}}{L_{\mathrm{s}}}\right),
$$

where the three fitting parameters are given by $r_{\mathrm{s}}=0.2 \mathrm{fm}, L_{\mathrm{s}}=68.7 \mathrm{MeV}$, and $\delta L_{\mathrm{s}}=6.8 \mathrm{MeV}$ represents the $70 \%$ prediction-band error. In particular, this suggests that a $\pm 0.06 \mathrm{fm}$ error in the measurement of $R_{\text {skin }}^{208}$ would translate into a $1 \sigma$ error in $L$ of $\Delta L(0.06 \mathrm{fm})=40.8 \mathrm{MeV}$. To properly estimate the final uncertainty in the determination of $L$, one should add both theoretical and experimental errors in quadrature.

We close this section with a brief comment on the frequently assumed correlation between $R_{\text {skin }}^{208}$ and the symmetry energy at saturation density $J=S\left(\rho_{0}\right)$. Whereas we have invoked robust, physical arguments in favor of a strong correlation between $R_{\text {skin }}^{208}$ and $L$, the correlation between $R_{\text {skin }}^{208}$ and $J$, or rather $J$ and $L$, has a different physical origin. Farine et al in 1978 first noted a correlation between $J$ and $L$ [48]. Indeed, it has been recognized for a long time that nuclear ground-state properties — especially nuclear masses of neutron-rich nucleidetermine rather accurately the value of the symmetry energy at a sub-saturation density $\rho \approx 0.1 \mathrm{fm}^{-3} \approx(2 / 3) \rho_{0}$, that represents an average between the central nuclear density $\rho_{0}$ and some characteristic surface density. In particular, [49] using the family of Skyrme CSkp functionals to fit ground-state properties of double magic nuclei found a value of $S\left(\rho=0.1 \mathrm{fm}^{-3}\right)=25.4 \pm 0.8 \mathrm{MeV}$. A comparable analysis by [50] using the masses of 38 spherical nuclei gives a value of the symmetry energy at a slightly higher density of $S\left(\rho=0.11 \mathrm{fm}^{-3}\right)=26.65 \pm 0.2 \mathrm{MeV}$. Given such an accurate determination of the symmetry energy at $\rho \approx(2 / 3) \rho_{0}$, the correlation between $J$ and $L$ is readily obtained from invoking equation (5). That is,

$$
S\left(\rho=2 \rho_{0} / 3\right)=J-\frac{L}{9}+\frac{K_{\text {sym }}}{162}+\cdots \approx J-\frac{L}{9} .
$$

Thus, the correlation between $J$ and $L$ emerges from the accurate determination of the masses of neutron-rich nuclei, rather than in the case of $R_{\text {skin }}^{208}$ and $L$ which arises from the competition between surface tension and the density dependence of the symmetry energy.

\subsection{Isoscalar monopole resonance}

The isoscalar monopole resonance measures the collective response of the nucleus to density variations. Pictorially, this collective excitation in which protons and neutrons oscillate 
in phase around the equilibrium density may be perceived as a nuclear breathing mode. Given that symmetric nuclear matter saturates, the pressure at saturation density vanishes. Thus, the giant monopole resonance (GMR) probes the curvature of the EOS at saturation density, or equivalently, the incompressibility coefficient of symmetric nuclear matter $K_{0}$. However, the accurate determination of $K_{0}$ requires the formation of a strong collective peak that involves many nucleons and exhausts most of the energy weighted sum rule. Thus, the coherent response of the system is sensitive to the incompressibility coefficient of neutron-rich matter $K_{0}(\alpha)$ rather than to only $K_{0}$; here $\alpha=(N-Z) / A$ is the neutron-proton asymmetry. As such, $K_{0}(\alpha)$ is sensitive to the density dependence of the symmetry energy. Indeed, the incompressibility coefficient of asymmetric nuclear matter may be written as follows [3]:

$$
K_{0}(\alpha)=K_{0}+K_{\tau} \alpha^{2} \equiv K_{0}+\left(K_{\mathrm{sym}}-6 L-\frac{Q_{0}}{K_{0}} L\right) \alpha^{2},
$$

where $Q_{0}$ is often referred to as the skewness parameter and is proportional to the third derivative with respect to the density of the energy per particle of symmetric nuclear matter evaluated at saturation density. That is,

$$
\mathcal{E}_{\mathrm{SNM}}=-a_{V}+\frac{1}{2} K_{0} x^{2}+\frac{1}{6} Q_{0} x^{3}+\cdots
$$

Note that the expression for $K_{0}(\alpha)$ given in equation (11) is exact to second order in $\alpha$. In principle, to constrain both $K_{0}$ and $K_{\tau}$ one would measure the distribution of monopole strength for nuclei with significantly different values of $\alpha$. In practice, however, access to the symmetry energy is hindered by the relatively low neutron-proton asymmetry of stable nuclei. Nevertheless, in an effort to determine the incompressibility of neutron-rich matter, a pioneering experiment was carried out at the Research Center for Nuclear Physics (RCNP) in Osaka, Japan [51, 52]. The experiment succeeded in measuring the distribution of isoscalar monopole strength in all stable Tin isotopes having an even number of neutrons, namely, from ${ }^{112} \mathrm{Sn}$ to ${ }^{124} \mathrm{Sn}$. Although the neutron-proton asymmetry along this isotopic chain varies from $\alpha=0.11-0.19$, the sensitivity to $K_{\tau}$ is poor-even for neutron-rich ${ }^{124} \mathrm{Sn}$. Although unrelated to the symmetry energy, the experiment uncovered a puzzle that remains to be solved: 'Why is Tin so soft?' [53]. That is, why do models that successfully reproduce GMR energies in ${ }^{90} \mathrm{Zr}$, ${ }^{144} \mathrm{Sm}$, and ${ }^{208} \mathrm{~Pb}$, overestimate the corresponding centroid energies along the full isotopic chain in $\mathrm{Sn}$.

\subsection{Electric dipole polarizability}

The oldest known and perhaps most prominent collective nuclear excitation is the isovector giant dipole resonance (GDR). This mode of excitation is perceived as an out-of-phase oscillation of neutrons against protons. Given that this oscillation results in the separation of two dilute quantum fluids - one neutron-rich and the other one proton-rich-the symmetry energy acts as the restoring force. The GDR is one state that contributes to the electric dipole polarizability. By using a covariance analysis with an accurately-calibrated density functional, it was recently demonstrated that the electric dipole polarizability $\alpha_{\mathrm{D}}$ is a strong isovector indicator that is highly correlated to the neutron-skin thickness of heavy nuclei [54]. Shortly after, using a large number of EDFs, it was confirmed that such a correlation is robustalthough some systematic model dependence emerged [55].

In an effort to elucidate the connection between the dipole polarizability and the density dependence of the symmetry energy, insights from the macroscopic liquid-droplet model are 

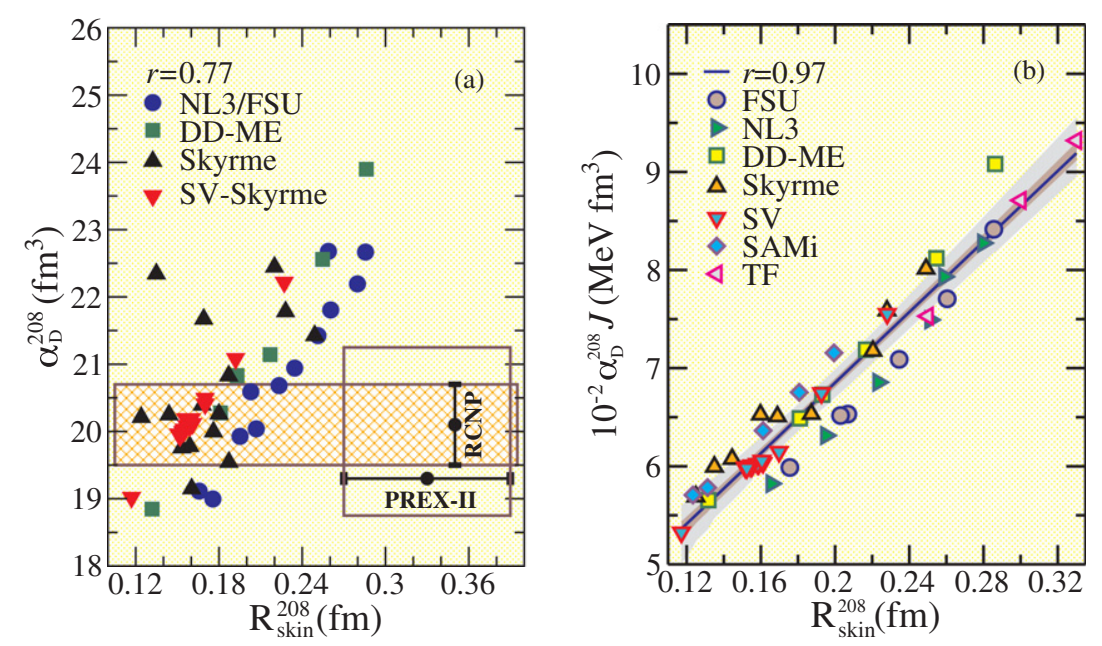

Figure 1. (a) Predictions from a large number of EDFs for the electric dipole polarizability and neutron-skin thickness of ${ }^{208} \mathrm{~Pb}$ as discussed in [55]. Constrains from both RCNP $[60,61]$ and the future PREX-II experiment (the latter assuming a projected $0.06 \mathrm{fm}$ error at the PREX central value [32]) have been incorporated into the plot. (b) Predictions from a large number of EDFs for $\alpha_{\mathrm{D}}^{208}$ times the symmetry energy at saturation density $J$ as a function of $R_{\text {skin. }}^{208}$. The lightly and darkly shaded regions in panel (b) represent the $99.9 \%$ and $70 \%$ confidence bands, respectively. These results were first reported in [59].

particularly helpful [56-59]. Within a droplet model ('DM') approach, the electric dipole polarizability takes the following simple form:

$$
\alpha_{\mathrm{D}}^{\mathrm{DM}} \approx \frac{\pi e^{2}}{54} \frac{A\left\langle r^{2}\right\rangle}{J}\left[1+\frac{5}{3} \frac{L}{J} \epsilon_{A}\right],
$$

where $\left\langle r^{2}\right\rangle$ is the mean-square radius of the nucleus and $\epsilon_{A}=\left(\rho_{0}-\rho_{A}\right) / 3 \rho_{0}$ accounts for the difference between the saturation density $\rho_{0}$ and an appropriate average density of the nucleus $A$ is $\rho_{A}$. To show the value of this qualitative formula in the particular case of ${ }^{208} \mathrm{~Pb}$, we display on the left-hand panel of figure 1 the correlation between $\alpha_{\mathrm{D}}^{208}$ and $R_{\text {skin }}^{208}$ as predicted by a large number of EDFs [55]. Although a clear correlation between the dipole polarizability and the neutron-skin thickness is observed, there is an appreciable scatter in the predictions; this yields a correlation coefficient of $r=0.77$. Note that by imposing the recent experimental constraint from $\alpha_{\mathrm{D}}^{208}[60,61]$ several models, especially those with a very stiff symmetry energy, may already be ruled out. Notably, if the follow-up PREX experiment (PREX-II) — with a projected uncertainty of $0.06 \mathrm{fm}$-finds that its central value of $R_{\text {skin }}^{208}=0.33 \mathrm{fm}$ remains intact, then all models displayed in the figure will be ruled out! [62]. Although provocative, we want to underscore that the above statement is contingent upon confirmation by PREX-II of PREX's large central value. At present, PREX with its large errors bars $\left(R_{\text {skin }}^{208}=0.33_{-0.18}^{+0.16} \mathrm{fm}\right)$ is unable to exclude the majority of models included in figure 1. Although the correlation displayed in figure 1(a) is evident, equation (13) suggests that the dipole polarizability times the symmetry energy at saturation density $\left(\alpha_{\mathrm{D}} J\right)$ should be far better correlated to $L$ (or equivalently to $R_{\text {skin }}^{208}$ ) than $\alpha_{\mathrm{D}}$ alone [59]. Thus, the right-hand panel of figure 1 displays $J \alpha_{\mathrm{D}}^{208}$ as a function of $R_{\text {skin }}^{208}$ as predicted by an augmented set of EDFs [59]. Remarkably, the fairly large spread in the model predictions has been practically eliminated by simply scaling $\alpha_{\mathrm{D}}^{208}$ by the $J$ associated to each model. With a correlation coefficient of $r=0.97$, this suggests that accurate measurements of both $R_{\text {skin }}^{208}$ and $\alpha_{\mathrm{D}}^{208}$ may provide stringent constraints on $J$ and $L$. Finally, we note that 
novel experimental techniques now provide access to other nuclear excitations-such as isovector-quadrupole and spin-dipole resonances - that appear to be sensitive to the density dependence of the symmetry energy. For a recent comprehensive review see [63].

\section{Symmetry energy at high densities in neutron stars}

The structure of spherical neutron stars in hydrostatic equilibrium is uniquely determined by the EOS of neutron rich matter, namely, the pressure as a function of energy density. The EOS depends on the interactions between nucleons and impacts nuclear-structure and heavy-ion observables. Therefore, a measurement in one domain-be it astrophysics or in the laboratory-can have important implications in the other.

\subsection{Neutron star radii}

Observations of neutron star masses and radii directly constrain the EOS. Indeed, a complete determination of the Mass-versus-Radius relationship will uniquely constrain the EOS [64]. Critically important to this task is the recent observation of a two solar mass neutron star by [65]. The determination of the stellar mass is very clean and accurate as it depends on the measurement of Shapiro delay in the pulsar's radio signal by the gravitational well of its white dwarf companion. Furthermore, the existence of massive neutron stars has now been confirmed with the discovery of a second two solar mass neutron star [66]. These observations have immediate and important implications: the pressure of matter at high densities must be sufficiently large to support a two solar mass neutron star against the gravitational collapse into a black hole. In particular these observations rule out many soft (low pressure) EOSincluding those containing hyperons or nearly free quarks and gluons. However, neutron stars could still harbor quark matter or hyperons in their cores provided the (poorly constrained) strong interactions among these exotic constituents can significantly increase the pressure at high densities.

Combined mass and radius estimates for several neutron stars have been inferred from observations of x-ray bursts [67-72]. X-ray bursts are thermonuclear explosion triggered by the accretion of matter from a companion unto the surface of the neutron star. In some bursts the luminosity becomes large enough to reach the Eddington limit, in which the photosphere is pushed outward from the radiative momentum flux. Thus, at the moment after the burst peak when the photosphere 'touches down', the flux observed by a remote observer at a distance $D$ should be

$$
F_{\text {Edd }, \infty}=\frac{G M c}{\kappa D^{2}}[1-2 \beta(R)]^{1 / 2} .
$$

Here $\beta(R)=G M / R c^{2}$ is the compactness, $M$ is the gravitational mass, $R$ is the local radius, i.e., $R=(2 \pi)^{-1} \times\{$ circumference of equipotential surface $\}$, and $\kappa$ is the opacity, which depends on the composition of the photosphere. The subscript ' $\infty$ ' denotes quantities measured by a distant observer.

In the latter, cooling, stage of the burst, the ratio of the observed flux $F_{\infty}$ to a fitted peak temperature $T_{\mathrm{bb}, \infty}$ is roughly constant. This ratio is therefore identified with the observed angular area of the neutron star,

$$
\frac{F_{\infty}}{\sigma T_{\mathrm{eff}, \infty}^{4}}=\left(\frac{R}{D}\right)^{2}[1-2 \beta(R)]^{-1} .
$$

Here $\sigma$ is the Stefan-Boltzmann constant, and the observed effective temperature $T_{\text {eff, } \infty}$ is related to $T_{\mathrm{bb}, \infty}$ via a composition-dependent correction factor $f_{c}$. 
Observations of both photospheric expansion and thermal cooling emission from a given system therefore allow a separate determination of both $R$ and $M$ from equations (14) and (15). Although in principle this is a powerful technique, there are important complications that may hinder the reliability of the method. For example, the correction $f_{c}$ required to model stellar atmospheres are large, presently uncertain, and may vary between different sources. Moreover, it is not yet clear from which X-ray burst systems, and at what time during the burst, one can extract the most accurate radii, nor is it entirely certain that the radii sampled by both observations are identical [69]. Improved models of x-ray bursts (e.g., [73]) may be able to address these uncertainties and allow for a more reliable determination of stellar radii.

Perhaps at present the cleanest way to extract neutron-star radii is from the observation of quiescent low mass x-ray binaries (qLMXB) in globular clusters. Such neutron stars are assumed to have accreted matter from a companion in the past, but are currently in a quiescent phase where they simply 'glow' in soft x-rays as they cool. Often they are assumed to have hydrogen atmospheres for which there are very accurate calculations of the emergent spectrum. The main argument in support of this assertion is that other heavier elements will rapidly sink in the very strong gravitational field and ultimately leave behind a hydrogen atmosphere [74]. Moreover, there is no evidence that these neutron stars are strongly magnetized, which makes the spectral models simpler. As a result, the observed effective temperature $T_{\text {eff, } \infty}$ can be determined directly by spectral fitting, which removes the systematic uncertainty in $f_{c}$, and the observed angular area can then be determined from equation (15). Although by itself this technique does not allow for independent determinations of $M$ and $R$ for a single source, observations of multiple stars-when combined with a constraint such as a functional form for the EOS [75] or an assumed dependence for $R(M)$ [76], permits the extraction of both. Although these measurements contain the distance $D$, one can use x-ray binaries in globular clusters - dense collections of up to a million stars - that have relatively well-established distances.

Recently [76] determined neutron-star radii from fitting the spectra of five qLMXBs with models appropriate for a nonmagnetic hydrogen atmosphere. Furthermore, they assumed that neutron-radii are approximately independent of mass, namely, $R(M)=R_{0}$. Indeed, such an assumption is consistent with many-although not all-models of the EOS over a wide range of masses [77]. By adopting such an assumption, [76] were able to fit the combined data sets with a single neutron-star radius,

$$
R_{0}=9.1_{-1.5}^{+1.3} \mathrm{~km}
$$

where the quoted errors are for the $90 \%$ confidence level. This fitting does not account for causality nor pulsar observations indicating that the maximum neutron star mass is greater than approximately $2.0 M_{\odot}[65,66]$; imposing these conditions as priors may change the result [78]. With these caveats, however, equation (16) is intriguing as it suggests stellar radii significantly smaller than those predicted by many theoretical models; at least models with non-exotic constituents [77]. Moreover, such a small radius coupled with the observation of neutron stars with $M \approx 2.0 M_{\odot}$ greatly constrains the EOS. On the one hand, the pressure near (2-3) $\rho_{0}$ must be small enough to accommodate small radii; on the other hand, the pressure must increase significantly at high densities in order to support massive neutron stars.

Unfortunately, there are important complications in applying the Stefan-Boltzmann law (equation (15)) to extract neutron-star radii. First, in order to determine the luminosity $L_{\infty}$ one needs an accurate distance to the star. Whereas globular clusters provide reliable distance estimates, systematic differences at the $\approx 20 \%$ level remain between various techniques (see [79] and references therein). Second, one must correct for interstellar absorption, which may be important in the far- ultraviolet and soft $\mathrm{x}$-ray energies. The systematic uncertainty 
associated to the distance measurement should be eliminated with the imminent launch of the Gaia spacecraft by the European Space Agency. Gaia will determine parallax angles (i.e., trigonometric distances) with an unprecedented accuracy of $2 \%$ (or better) for millions of stars on these globular clusters (see [80], and references therein). However, relying on the Stefan-Boltzmann law assumes that neutron stars may be modeled as black bodies-which in general they are not. Indeed, one must fit the observed spectrum to a model atmosphere that depends on the composition and perhaps on the stellar magnetic field. In addition, by using a single effective temperature $T_{\mathrm{eff}, \infty}$ in equation (15) one assumes spherical symmetry. If the temperature distribution is anisotropic, however, (for example from anisotropic heat conduction in a strong magnetic field) then the extracted stellar radius may be inaccurate. Note that there is no evidence for a strong magnetic field and pulsations in the x-ray flux are not observed.

Perhaps the simplest way to accommodate neutron stars with small radii as in equation (16) is for the EOS to be soft up to densities of $\rho \lesssim(2-3) \rho_{0}$, (see [69], and references therein). This would suggest that the neutron skin-thickness of ${ }^{208} \mathrm{~Pb}$ should also be small-a fact that can be directly tested via parity violating electron scattering at Jefferson Lab; see section 4.1. Alternatively - and more intriguing - the pressure at low densities could be large, leading to a thick neutron skin in ${ }^{208} \mathrm{~Pb}$. This would require a rapid softening of the EOS in order for the pressure at (2-3) $\rho_{0}$ to be small enough to accommodate equation (16). Such rapid density dependence could come from a change in the structure of dense matter, possibly from a phase transition, that could be probed with heavy-ion collisions. Note that regardless of the softening mechanism, the EOS must stiffen again at higher densities in order to support massive neutron stars.

In summary, the following three observables are sensitive to the EOS at different densities. First, the neutron-skin thickness of ${ }^{208} \mathrm{~Pb}$ is sensitive to the pressure at $\rho_{0}$ and below. Second, the radius of an $1.4 M_{\odot}$ neutron star is sensitive to the pressure over a range of densities, but is most sensitive to densities in the range (2-3) $\rho_{0}$ [81]. Finally. the maximum mass of a neutron star is particularly sensitive to the pressure at high densities. Therefore, the density dependence of the EOS can be deduced by comparing these three observables.

\subsection{Neutron star cooling}

Modeling of cooling neutron stars [82] predated their first detection. For an exhaustive review of neutron star cooling, see [9]; here we just give a summary of the salient features involving the nuclear symmetry energy. The core of a neutron star is in $\beta$-equilibrium. To maintain this equilibrium, the following reactions are in balance:

$$
\begin{aligned}
& \mathrm{n} \rightarrow \mathrm{p}+\mathrm{e}^{-}+\bar{v}_{e} \\
& \mathrm{p}+\mathrm{e}^{-} \rightarrow \mathrm{n}+v_{e} .
\end{aligned}
$$

For degenerate npe $\mathrm{e}^{-}$matter, integration of the cross-section over the available phase space gives a characteristic $T^{6}$ temperature dependence to the neutrino emissivity, known as the direct Urca (dUrca) process. The reactions (17) and (18) cannot, however, simultaneously satisfy momentum and energy conservation unless the proton fraction $x \equiv \rho_{\mathrm{p}} /\left(\rho_{\mathrm{p}}+\rho_{\mathrm{n}}\right)$ is greater than approximately 0.11 [83]. As a result, for sufficiently low proton fraction the reaction proceeds via a bystander particle (for example),

$$
\begin{aligned}
& \mathrm{n}+\mathrm{n} \rightarrow \mathrm{n}+\mathrm{p}+\mathrm{e}^{-}+\bar{v}_{e} \\
& \mathrm{n}+\mathrm{p}+\mathrm{e}^{-} \rightarrow \mathrm{n}+\mathrm{n}+v_{e},
\end{aligned}
$$

and similar reactions for which the bystander particle is a proton. These reactions have a $T^{8}$ temperature dependence and have a much lower rate at temperatures $\lesssim 0.1 \mathrm{GK}$, which are typical core temperatures for observed cooling neutron stars. 
The pressure of nuclear matter at densities near saturation is proportional to $\partial S / \partial \rho$. If this term increases rapidly with density, then it is energetically favorable for the proton fraction to be large. Moreover, if this trend holds at larger densities, then the interior of the neutron star may reach a proton fraction $x>0.11$ before the star reaches its maximum mass. If so, then there is a neutron star mass threshold $M_{\mathrm{dUrca}}$ above which direct Urca reactions (equations (17) and (18)) can occur. To date, all observed isolated cooling neutron stars are consistent with standard (i.e., no enhanced or dUrca) cooling [84]). Note that enhanced cooling could make isolated neutron stars so cold and dim that they are not observed. Indeed, there are many supernova remnants with no detected central compact object $[85,86]$. While it is perhaps unlikely that all of the missing sources could be black holes, our knowledge of stellar evolution and collapse is insufficient to draw firmer conclusions.

Quiescent neutron stars in transient low-mass X-ray binaries (qLMXBs) offer another test for the presence of enhanced cooling. When the neutron star accretes, compression of matter in the crust raises the electron and neutron chemical potentials, which induce nuclear reactions [87-89] that in turn heat the neutron star crust and core. The crust and core temperatures come into equilibrium [90] in which the heat deposited in the crust is radiated via neutrinos from the core or photons from the surface during quiescent periods when the accretion rate is very low or zero. Observations of qLMXBs can therefore inform us about the interior temperature and the strength of neutrino emission in the neutron star [91].

Most observed qLMXBs are consistent with having standard cooling [92], given the large uncertainties in the long-term mean-mass accretion rates. However, there are two notable exceptions. The first is SAX J1808.4-3658, which is a pulsing, accreting neutron star. For this source only an upper limit on the thermal component of the luminosity is reported, at $1.1 \times$ $10^{31} \mathrm{erg} \mathrm{s}^{-1}$ [92]. This implies a core temperature (20-30)MK [93]. If this core temperature is set by balancing accretion-induced heating with neutrino cooling then the presence of enhanced cooling is required. An even colder neutron star is in the transient $1 \mathrm{H} 1905+00$ for which only an upper limit of $2.4 \times 10^{30} \mathrm{erg} \mathrm{s}^{-1}$ on the luminosity is reported [94]. For this system, the time-averaged mass accretion rate is unknown, unfortunately, so more quantitative evaluations of the neutrino cooling are not possible.

If enhanced cooling is not observed, this suggests that the symmetry energy at high densities is not large so that dUrca is not possible. Furthermore, the beta decays of additional hadrons such as hyperons, if present, are suppressed (for example by large pairing gaps). Alternatively, if enhanced cooling is confirmed, in at least some neutron stars, then one can constrain the particles responsible for the cooling. Determining the high density symmetry energy from heavy ion collisions, section 6, can rule in, or out, dUrca. If dUrca is ruled out, this suggests the enhanced cooling is from the beta decay of other strongly-interacting particles such as hyperons, mesons, or quarks. This would demonstrate that dense matter contains other constituents in addition to just neutrons and protons.

\section{The symmetry energy and heavy-ion collisions}

Heavy-ion collisions provide the only means to investigate the behavior of the symmetry energy at high densities within a laboratory environment by compressing nuclear matter from moderate to high densities. At low incident energies-from 30 to $150 \mathrm{MeV}$ per nucleon-expansion after the initial compression creates a low density region where symmetry-energy effects including cluster formation can be studied. The forces resulting from such a compression and the subsequent expansion influence strongly the motion of the ejected matter and provide observables that are sensitive to the EOS. Extracting information of relevance to the symmetry energy presents a serious challenge given the relatively low neutron-proton asymmetry of the 
colliding ions. To overcome this obstacle one makes use of the fact that the forces associated with the symmetry energy affect differently the various members of an isospin multiplet. Enhanced sensitivity to the density dependence of the symmetry energy is then obtained by comparing neutron- against proton-like observables. As full equilibrium is never reached in heavy-ion collisions, experimental analyses depend heavily on theoretical transport models.

The dynamical theories underlying these transport models rely on various assumptions and approximations. For the most part these models are based either on a semiclassical approximation to the time-dependent Hartree-Fock equations (Boltzmann- UehlingUhlenbeck (BUU)) or on molecular dynamics simulations that incorporate quantum effects (such as quantum molecular dynamics (QMD) and antisymmetrized molecular dynamics (AMD)). These models include a mean-field potential usually with momentum dependence plus a collision term with in-medium NN cross sections, which describes the dissipative features of the collision. The mean field also includes a 'tunable' density dependence for the symmetry energy. Since the transport equations are not solvable directly, they are treated by simulations. Given their critical role in the interpretation of heavy-ion collisions, we provide in a separate subsection (section 6.3) a brief description of transport models, their current development, and ways to improve the consistency and reliability of their predictions.

Investigations on the EOS of symmetric nuclear matter have been very successful in understanding a wide set of observables - such as flows of nucleons and light particles, and kaon production [95, 96] - from transport-model predictions. These efforts have provided significant constraints on the EOS of symmetric nuclear matter. A similar comprehensive effort is now needed to place more stringent constraints on the density dependence of the symmetry energy. Consequently heavy-ion collisions have increasingly been used in recent years to probe the symmetry energy over a wide range of densities by varying the incident energy, impact parameter, and isospin asymmetry of the colliding system. In the following sections observables used to probe the symmetry energy are introduced, first at sub-saturation densities, followed by a discussion of the observables proposed for super-saturation densities. In each density domain, difficulties in the theoretical description of these observables are identified and strategies to clarify them and improve their predictive power are discussed.

\subsection{Heavy-ion collisions at low densities}

Heavy-ion collisions at incident energies from about 35 to $150 \mathrm{MeV}$ per nucleon give access to the symmetry energy at densities from about $50 \%$ above $\rho_{0}$ down to about $0.1 \rho_{0}$. The initially compressed nuclei expand toward low densities where many intermediate mass fragments are formed. Nucleons and light clusters such as deuterons, tritons, and alpha particles are emitted during the fragmentation process as well as from the excited primary fragments. By selecting collision geometries from peripheral to central collisions, one can study a number of phenomena which depend on the transport of isospin and thus provide information on the density dependence of the symmetry energy.

In semi-peripheral collisions the amount of isospin diffusion $(N / Z$ equilibration) between collision partners of different neutron-proton asymmetries is driven by the symmetry energy at subsaturation densities. The degree of isospin diffusion is quantified in terms of an isospin transport ratio by comparing reaction systems with different combinations of neutron-rich and neutron-poor projectiles and targets. For example, by using heavy-ion collision data of ${ }^{112} \mathrm{Sn}$ and ${ }^{124} \mathrm{Sn}$ in identical and mixed combinations at $50 \mathrm{MeV}$ nucleon ${ }^{-1}$, constraints on the symmetry energy were obtained for densities in the range $\rho=\rho_{0} / 3$ to $\rho_{0}$ using several isospin diffusion observable [97] under the assumption that clusters do not affect the symmetry energy in the region where isospin diffusion occurs. 

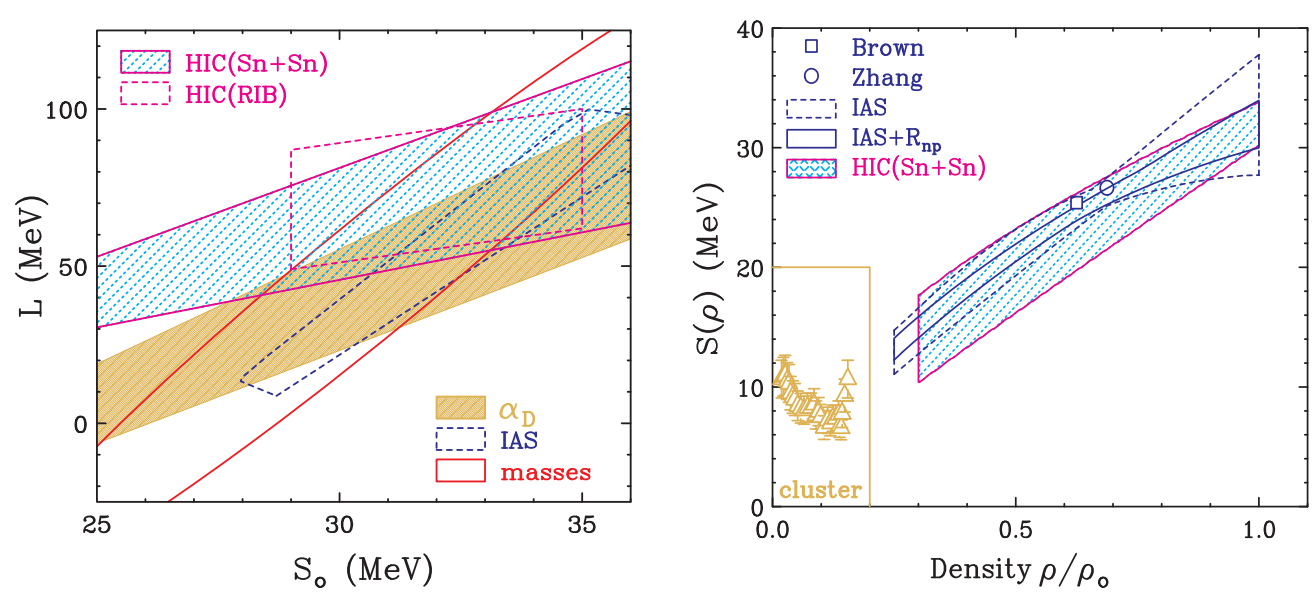

Figure 2. Constraints on the density dependence of the symmetry energy from both heavy-ion collisions and nuclear-structure observables. Left: correlation between $S_{0}$ and the slope of the symmetry energy $L$ at saturation density (see text for a detailed description). Right: the symmetry energy $S(\rho)$ as a function of baryon density (see text for a detailed description).

Constraints on the density dependence of the symmetry energy obtained from heavy-ion experiments are shown in figure 2 in two representations and are compared against those obtained from nuclear-structure observables discussed in section 4. On the left-hand panel of figure 2 we display constraints on the symmetry energy $S_{0} \equiv J$ and its slope $L$ at saturation density. The blue hatched area labeled $\mathrm{HIC}(\mathrm{Sn}+\mathrm{Sn})$ was determined from isospin diffusion observables measured in mid-peripheral collisions of Sn isotopes [97]. A constraint on the symmetry energy obtained in recent measurements of the mean $N / Z$ distributions of the emitted fragments with radioactive ion beams of ${ }^{32} \mathrm{Mg}$ on a ${ }^{9} \mathrm{Be}$ target at $73 \mathrm{MeV}$ per nucleon is shown by the area enclosed by the dashed purple line labeled HIC(RIB) [98]. (Note, that the limits of $S_{0}$ in these two areas only indicate the range of values used in the transport simulations and are not to be interpreted as experimental limits on $S_{0}$ from HICs.) These constraints from HICs are compared against those obtained from nuclear structure; in particular, from studies of (a) isobaric analogue resonances (blue dashed polygon) [99], (b) the electric dipole polarizability in ${ }^{208} \mathrm{~Pb}$ (gold shaded region) $[59,60,100]$ both with better than $90 \%$ confidence limit $(\mathrm{Cl})$, and (c) nuclear binding energies using the UNEDFO EDF (two red curves forming part of an ellipsoid, about $90 \% \mathrm{Cl}$ ) [101]. These are basically independent constraints, and one may get a stronger constraint in principle by finding the overlap region in the plane of $S_{0}$ and $L$. However, for each of these constraints, we observe a strong correlation between $S_{0}$ and $L$, suggesting that the observable is sensitive to the symmetry energy at low densities.

As alluded in section 4.1, the masses of neutron-rich nuclei place a stringent constraint on the value of the symmetry energy around $\rho=(2 / 3) \rho_{0}$; see equation (10). In particular, two analyses with Skyrme functionals found the rather tight values of $S\left(\rho=0.1 \mathrm{fm}^{-3}\right)=$ $25.4 \pm 0.8 \mathrm{MeV}$ [49] and $S\left(\rho=0.11 \mathrm{fm}^{-3}\right)=26.65 \pm 0.2 \mathrm{MeV}$ [50]. These values have been plotted in the right-hand panel of figure 2 as an open square and an open circle, respectively.

On the right-hand panel of figure 2 we show a different interpretation of the constraints by focusing directly on the density dependence of the symmetry energy $S(\rho)$. The shaded area labeled $\mathrm{HIC}(\mathrm{Sn}+\mathrm{Sn})$ results from the analysis also shown on the left of isospin diffusion observables from [97]. From the analysis of isobaric analogue states (IAS) by [99] two constraints have been reported. The area enclosed by the dashed blue line comes from the 
same IAS analysis shown on the left-hand panel. The area enclosed by the solid blue line results when the IAS analysis is supplemented with additional constrains from neutron-skin data $R_{\text {skin }}$. Note that the addition of neutron-skin information reduces significantly the constraint areaespecially near saturation density. This is natural given the strong correlation between $R_{\text {skin }}$ and the slope of the symmetry energy $L$, noted earlier. However, one should remember that the extraction of $R_{\text {skin }}$ from hadronic experiments is beset by large theoretical uncertainties.

The symmetry energy at very low density obtained from the analysis of cluster production in HICs as discussed in section 3 is shown enclosed by a box in the right-hand panel of figure 2 . Here it should be noted that this analysis determines the symmetry energy with strong cluster correlations at a temperature of several $\mathrm{MeV}$, depending on the density. Due to the large temperature dependence of the symmetry energy at very low density, it cannot be compared directly to the symmetry energy at higher densities.

Altogether we see from figure 2 that heavy-ion results are consistent with those extracted from nuclear-structure information. This lends credibility to the use of heavy-ion collisions as a probe of the symmetry energy at supra-saturation densities, a region inaccessible to nuclear-structure experiments (see section 6.2).

In central collisions of neutron-rich systems one observes isospin fractionation, namely, a differentiation of the isospin distribution among the reaction products [102, 103]. In a neutronrich environment the neutron mean-field potential is more repulsive than that of the proton. This enhances the neutron emission in such a way that the neutron-to-proton ratio of the emitted particles (gas) is larger than that of the formed fragments (liquid). Then, the spectra and the spectral ratios of emitted particles should be a direct probe of the symmetry energy. The experimental ratios at low energies in the center-of-mass system are large, much beyond Coulomb effect expectations. However, a complete understanding of this phenomenon has not yet been achieved because of two aspects of the transport models. The first one is associated with the isovector momentum dependence-also expressed as the neutron-proton effective mass difference-which is highly uncertain and influences the high-energy part of the particle spectra. The second one involves the treatment of cluster formation which affects the lowenergy particle spectra. As discussed in section 3 and in [104], cluster formation increases the symmetry energy at very low densities and may affect neutrino transport in the neutrinosphere. Cluster formation is important in heavy-ion collisions-especially in the expansion phase. For example, in the final state of $\mathrm{Sn}+\mathrm{Sn}$ collisions at $50 \mathrm{MeV}$ nucleon ${ }^{-1}$, about $90 \%$ of the protons in the system are bound in clusters and heavier fragments. However, the treatment of cluster formation in transport theories, including how to take into account the physical level density of the in-medium clusters which may contain bound and resonance states, is a difficult problem, which will be discussed more in section 6.3.

Neutron detection efficiencies are often uncertain, thus ratios of $n / p$ ratios, i.e. double ratios, of systems with different asymmetry have been obtained, which are hopefully less affected by the neutron efficiency, but some of the sensitivity of the single $n / p$ ratio may be reduced in the double ratios. Alternatively, one has measured $t /{ }^{3} \mathrm{He}$ ratios, which display similar trends as the $n / p$ ratios. However, their theoretical description requires a better description of the cluster production mechanism. For example, a recent theoretical and experimental study finds that the formation of alphas is important in describing the triton to ${ }^{3} \mathrm{He}$ spectra and spectrum ratio. The problem of describing cluster formation can be somewhat alleviated by constructing 'coalescence invariant' quantities, i.e., observables summed over all light clusters, which show much better agreement between theory and experiment $[105,106]$.

Furthermore intermediate mass fragments are copiously emitted in lower energy heavy-ion collisions, which is a process not only interesting in itself but which also contains information on the symmetry energy. It has been shown $[107,108]$ that different reaction mechanisms from 
isospin fractionation in the disintegrating system to isospin migration to the low-density neck region, as well as cluster correlations, depend on the density-dependence of the symmetry energy $[105,109,110]$.

In the current situation, possible ways to go forward are (1) to find observables which can be predicted with minimum uncertainty from cluster production by available transport models, (2) to compare the predictions of different models in detail until the physical origins of different predictions become clear (see subsection on transport models, section 6.3), (3) to establish improved models that can consistently describe the global reaction dynamics (including cluster and fragment formation) together with the observables sensitive to the symmetry energy. Efforts for solutions to the options (2) and (3) are particularly important. Specifically, models should be able to describe many experimental observables simultaneously.

On the experimental front, measurements with larger isospin asymmetry made possible with the availability of high intensity rare isotope beams provide higher sensitivities to the symmetry energy and will be able to further clarify these issues and establish the understanding of particle emissions in neutron-rich systems. An experiment that measures isospin diffusion using radioactive beams of ${ }^{109}$ In on a ${ }^{124} \mathrm{Sn}$ target $(\Delta(\delta)=0.093)$ has recently been performed at RIKEN. A more desirable reaction is to use a radioactive ${ }^{132} \mathrm{Sn}$ beam on a ${ }^{112} \mathrm{Sn}$ target $(\Delta(\delta)=0.135)$. By increasing the asymmetry by more than $50 \%$, improved uncertainty limits of the constraints are expected.

\subsection{Heavy-ion collisions at higher densities}

Although there has been enormous progress in elucidating the nature of cold hadronic matter at very high densities directly from QCD [111], the density region where these predictions apply is out of reach of experimental tests in high energy heavy-ion collisons and even at the enormous densities that exist in the core of neutron stars. Hence, for these density regions one must rely on theoretical models that, unfortunately, differ dramatically in their prediction of the high-density behavior of the EOS [112]. A major source of these difficulties is the incomplete knowledge of the short range isovector correlations [113]. In order to constrain the models one must rely on both laboratory experiments with energetic heavy ions and astronomical observations of neutron stars.

Observations of massive neutron stars $[65,66]$ imply that the high-density component of the EOS must be stiff. The recent analysis by [76] seems to suggest that neutron stars have small radii, implying a rather soft symmetry energy in the region of $\rho=(2-3) \rho_{0}$ (see section 5). In combination, both of these results-large stellar masses and small radii-pose serious challenges to theories of hadronic matter without exotic degrees of freedom.

Intermediate and high-energy (relativistic) heavy-ion collisions with rare isotope beams can be used to study neutron-rich systems that attain this range of densities for short-time intervals and may help elucidate the nature of these puzzles. At collision energies from about a hundred $\mathrm{MeV}$ to a few $\mathrm{GeV}$, nuclear matter is compressed from about 1.5 up to $3.5 \rho_{0}$ in a short time interval of about (10-100) $\mathrm{fm} \mathrm{c}^{-1}$ or about $10^{-22} \mathrm{~s}$. For example, the top panel of figure 3 shows the time evolution of the central density in a $\mathrm{Au}+\mathrm{Au}$ collision at $1 \mathrm{GeVu}^{-1}$, suggesting that nucleonic matter may be compressed to almost $3 \rho_{0}$ for a period of about $(5-10) \mathrm{fm} \mathrm{c}^{-1}$.

The big challenge to theoretical descriptions of heavy-ion collisions is the extraction of properties relevant to the high-density phase from the experimental observables constructed from the particles detected in the final stages of the reactions. As in the case of low-density matter, the aim is to magnify the impact of the symmetry energy by measuring differences or ratios of observables involving charge-symmetric pairs of particles, such as $n / p, t /{ }^{3} \mathrm{He}$ or 


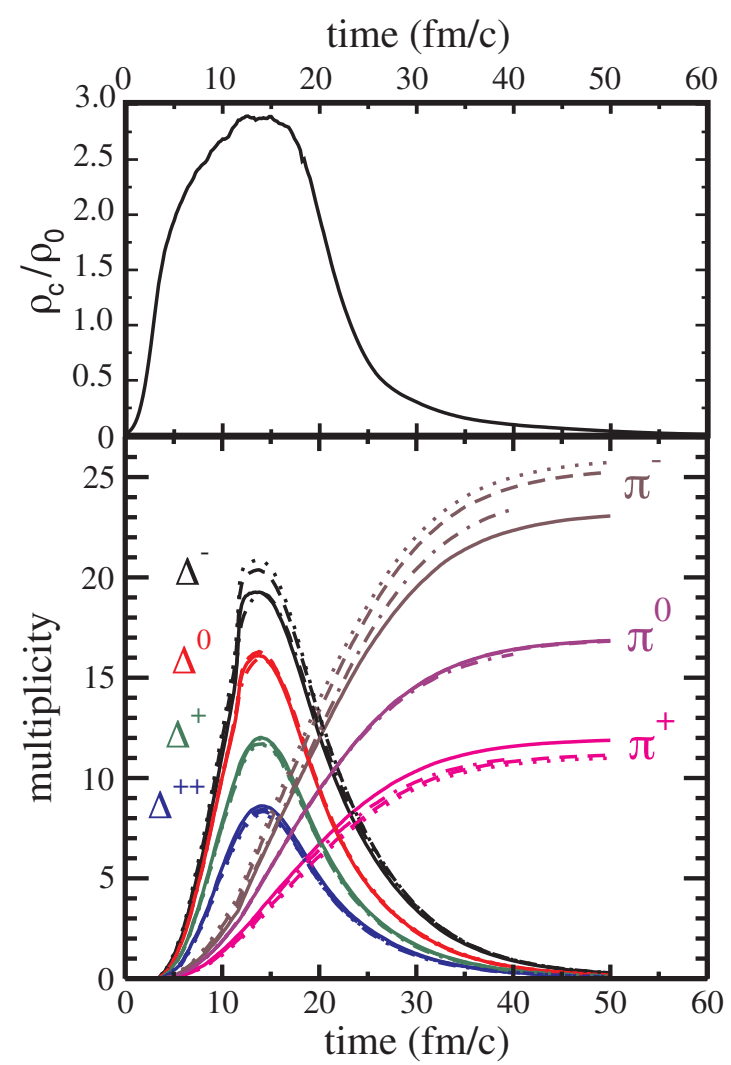

Figure 3. Time evolution of a central $\mathrm{Au}+\mathrm{Au}$ collision at en energy of $E=1 \mathrm{GeVu}^{-1}$. Top panel: density in the central cell in units of saturation density $\rho_{0}$. Bottom panel: multiplicities of produced $\Delta^{(-, 0,+,++)}$ and $\pi^{(-, 0,+)}$ particles. The curves correspond to calculations with different assumptions on the symmetry energy: stiff (dotted), linear (dashed), soft (dot-dashed), only kinetic symmetry energy (solid) [114].

$\pi^{-} / \pi^{+}$. In anticipation of these critical experiments, at least two time projection chambers (TPC) are being commissioned: the active target time projection chamber (AT-TPC) at NSCL/FRIB and the SAMURAI-TPC detector at RIKEN, where an experimental program involving central collisions of ${ }^{132} \mathrm{Sn}+{ }^{124} \mathrm{Sn}$ and ${ }^{124} \mathrm{Sn}+{ }^{112} \mathrm{Sn}$ at 200 and $300 \mathrm{MeV}$ per nucleon is being planned.

There already exist some data and corresponding transport-model predictions for flow and pion ratio observables, but the available experimental and theoretical information is far from complete. The existing data (from the Kaos and FOPI collaborations at GSI) involve nucleon, light cluster, and pion observables measured mainly in $\mathrm{Au}+\mathrm{Au}$ reactions at energies between $400 \mathrm{MeV}$ and $1.5 \mathrm{GeV}$ per nucleon $[115,116]$. These data were primarily obtained and analyzed for investigating the EOS of symmetric matter. However, the scarcity of neutron data and the lack of projectile-target systems with a wide range of neutron-proton asymmetries limits the sensitivity of these data to the symmetry energy. New measurements have been and will continue to be performed to overcome these limitations. Beams of widely different asymmetries from advanced rare isotope facilities will play a pivotal role in future studies by providing selective sensitivity to the symmetry energy while minimizing variations in the contributions from the symmetric matter EOS. In addition to studying the collisions of Sn 
isotopes including unstable neutron rich (e.g., ${ }^{132} \mathrm{Sn}$ ) and neutron poor (e.g., ${ }^{108} \mathrm{Sn}$ ) beams, a program is being developed to measure reaction systems with the same mass, such as ${ }^{112} \mathrm{Sn}+{ }^{112} \mathrm{Sn}$ and ${ }^{112} \mathrm{Ru}+{ }^{112} \mathrm{Sn}$ and highly asymmetric systems such as ${ }^{36} \mathrm{Ca}+{ }^{112} \mathrm{Sn}$ and ${ }^{52} \mathrm{Ca}+{ }^{124} \mathrm{Sn}$. These very asymmetric reactions provide a larger lever arm in the determination of critical isospin-dependent inputs for transport models, such as the symmetry potentials and the in-medium NN collisions cross sections.

Similarly as at lower densities, primary discriminators of symmetry-energy effects are expected to involve differences in neutron and proton observables, such as spectra and spectral ratios of emitted neutrons and protons or $t$ and ${ }^{3} \mathrm{He}$, as well as flow differences. The differences in neutron and proton potentials including the momentum dependence or the effective mass difference influence the asymmetry of the flow and the production of secondary particles. Some calculations suggest that at higher densities ratios of isospin yields and flows may be more sensitive to the nucleon mass splitting than to the density dependence of the symmetry energy [117]. If so, this provides an opportunity to determine separately both the momentum dependence of the interaction and the density dependence of the symmetry energy at high densities.

The collective motion induced by the pressure developed in the compressed region is quantified in terms of a Fourier series of the azimuthal distribution, where the first two coefficients are known as the transverse and elliptic flow. Of these, the elliptic flow probes the emission perpendicular to the reaction plane, and is therefore sensitive to pressure gradients in the high-density region of the collision. Thus, differences (or ratios) of the neutron-proton elliptic flow are regarded as sensitive probes of the high-density component of the symmetry energy. Indeed, there are indications that the elliptic-flow ratio of neutrons to protons is sensitive to the symmetry energy at supra-normal densities from the analysis of FOPI/LAND experiments at GSI [118, 119].

In high-energy collisions new particles are copiously produced. In particular, the ratio of negatively to positively charged pions $\pi^{-} / \pi^{+}$has been proposed as a probe of the symmetry energy at high densities $[120,121]$. Two estimates of pion production-the isobar model and the assumption of chemical equilibrium - both predict a strong sensitivity to the asymmetry of the medium and thus to the symmetry energy. In heavy-ion collisions pions are primarily produced in $\mathrm{NN}$-collisions via the excitation and subsequent decay of the $\Delta$-resonance. If the medium is neutron rich, the more abundant $n n$ collisions will increase the production of $\Delta^{0}$ and $\Delta^{-}$resonances, leading ultimately to an enhanced $\pi^{-} / \pi^{+}$ratio. For example, the excitation of the $\Delta^{-}$resonance and its ensuing $n \pi^{-}$decay is driven exclusively by the $n n \rightarrow p \Delta^{-}$ reaction and thus should significantly enhance the $\pi^{-} / \pi^{+}$ratio in a neutron-rich medium. Some theoretical analyses moreover predict that the mean-field effects and the threshold effects in the production of the particles influence the ratio in opposite directions, making predictions more critical [120]. Thus, imposing meaningful constraints on the symmetry energy depends crucially on a good understanding of the $\pi-\Delta$ dynamics in the medium. This constitutes a serious theoretical challenge, as, e.g., one must understand how the pion and $\Delta$ propagators (mass and width) are modified in the nuclear medium. Moreover, the propagation of particles with finite width has to be approximated. It has been noted that the sensitivity of pion production to the symmetry energy increases at lower incident energies where the in-medium masses of nucleons, Deltas, and pions (i.e. the real parts of their self-energies) provide critical threshold effects for particle production. In the bottom panel of figure 3 we display the time evolution of both the $\Delta$ and pion multiplicities. Comparing to the upper panel it is seen that Deltas exist mainly during the dense phase of the collision, while the pion multiplicities change also during the expansion phase due to reabsorption and charge exchange scattering. 
At present it seems that the different transport codes make different assumptions about the relevant self-energies for nucleons, Deltas, and pions as well as for the elastic and inelastic cross sections. This may contribute to the large differences in the model predictions of the pion ratio [120-123]. Currently, it is not clear whether a stiff or a soft symmetry energy provides a better description of the data. Indeed, in the calculations of [121] a very soft symmetry energy was postulated for the explanation of the experimental data, in strong disagreement with the conclusions from flow measurements [118]. Clearly, the current situation needs to be clarified. There is also a need for more data on pion spectra and pion flow for systems with fixed total charge and different asymmetry - to clearly distinguish between Coulomb and symmetry-energy effects. Such experiments will provide valuable constraints on transport models and should help to understand the situation. Eventually, pion yields should become a very useful source of information on the symmetry energy.

Pions are created and reabsorbed via the formation of $\Delta$ resonances. Reabsorption and pionic charge exchange increase with energy, and thus decrease the sensitivity to the high density phase of the collision, as seen in the bottom panel of figure 3. In this respect the investigation of kaon production could be advantageous and has been proposed as a sensitive probe of the symmetry energy [120]. In contrast to pions, $K^{0}$ and $K^{+}$mesons-which carry a strange antiquark-interact weakly with matter and have rather long mean-free paths and should therefore be good probes of the high density phase of the collision. In fact, the ratio of $K^{+}$mesons produced in collisions of light to heavy nuclei has provided in the past a robust observable for the stiffness of the EOS for symmetric matter [96]. Similarly, the ratio of $K^{0}$ (produced mainly from $n n$ collisions) to $K^{+}$(produced mainly from $p p$ collisions) has been predicted to be sensitive to the high-density behavior of the symmetry energy [114]. As in the case of pion production, the strongest sensitivity is expected near the kaon-production threshold, as many kaons are produced via pion-baryon reactions in the nuclear medium. To isolate symmetry-energy effects, the density and isospin dependence of the kaon self-energy must be properly understood [124]. Past measurements [125] suffered from very different acceptances for $K^{0}$ and $K^{+}$, requiring double ratios, but high-statistics measurements may now be possible with the HADES detector at GSI.

\subsection{Transport codes}

Transport models follow the evolution of the colliding system under the influence of meanfield potentials and of dissipation (encoded in a collision term). These models have been very successful for many years in both predicting and explaining many phenomena in heavy-ion reactions. However, in spite of this success questions remain-partly related to the formulation and partly to the implementation of the models. Given the critical role that transport models have in interpreting the experimental results of heavy-ion collisions, a way forward in the study of the symmetry energy requires an increase in the predictive power and reliability of transport codes. In this subsection we address some of these difficulties and propose ways to overcome them.

Different transport approaches have been developed over the years. Boltzmann-like approaches, known, e.g., under the names of BUU or stochastic mean field, describe the evolution of the single particle phase space density. In principle, they have to be augmented by a fluctuation term, leading to the Boltzmann-Langevin equation; various attempts in this directions have been put forward (see [126], and references therein). On the other hand, molecular dynamics approaches start from classical molecular dynamics and introduce finite size wave-packets, usually without anti-symmetrization (QMD). Collisions between these wave-packets lead to dissipation and to fluctuations which are controlled by the width of 
the particles. An significant further development is AMD (and approximately constrained molecular dynamics), which includes anti-symmetrization between the wave packets, and thus represents a quantum-mechanical transport model. The essential difference between Boltzmann and molecular dynamics approaches is the amount of fluctuation introduced into the evolution of the system. In general, fluctuations are important when the evolution enters a spinodal region of the phase diagram, where the phase transition leads to the formation of fragments or light clusters. This is very important in collisions in the low density region (see subsection 6.1) but also at higher densities clusters constitute an essential fraction of the ejectiles. A reliable description of cluster production is therefore crucial in the comparison with experiments.

The effect of cluster formation on the reaction dynamics and of isospin migration driven by both density and isospin gradients has been studied using a version of the BUU approach that treats clusters (deuterons, tritons, and ${ }^{3} \mathrm{He}$, but not alpha particles) as distinguishable degrees of freedom [105]. The collision terms couple the equations for the different particles. In the QMD and AMD approaches which employ nucleon wave packets, a cluster can be naturally described by placing the appropriate wave packets at the same phase space point. However, the probability of forming a cluster is then governed by the classical phase space in which the quantum bound state or resonance contribution is missing. In a recent version of AMD, the collision term has been improved in order to better incorporate the probability that one (or both) of the colliding nucleons form a cluster (with $A=2,3$ and 4) with other particles in the system $[127,128]$. Care should be taken, that when light clusters are treated as new degrees of freedom in the transport approach, their effect should not also be included into the parameterization of the EOS, particularly not in the symmetry energy. Both BUU and AMD calculations including clusters demonstrate that cluster formation can change the reaction dynamics and fragmentation mechanism [105, 127, 128]. This effect may explain the differences in the isospin transport ratios obtained with usual BUU calculations [129] relative to QMD calculations which have different cluster formation probabilities [97].

The dynamical evolution of particles with finite width ('off-shell transport') is in principle understood in the framework of the Kadanoff-Baym equations, but usually neglected or treated in simple approximations. It is expected, however, to be important in the sub- or near-threshold production of particles, like pions, $\Delta$-resonances, or strange particles, which are important probes of the symmetry energy at higher density.

The physical input into transport simulations are the mean-field potential, often derived from an EDF, and the in-medium elastic and inelastic cross sections. Both of these are often parametrized independently of each other, even though, in principle, they are related by a consistent approximation to the in-medium effective $T$-matrix, e.g., in the Brueckner-HartreeFock approximation. A consistent approach in all models is a highly desirable goal. We note that an important check involves solving the transport models in a confined box to simulate the conditions of thermally equilibrated systems. This can test the relation between the adopted effective interaction and the corresponding EOS [130-132]. Moreover, it can provide useful links to dynamical conditions, such as those found in the core and crust of neutrons stars and in supernovae explosions.

In all approaches, the set of transport equations constitutes a complex system of nonlinear integro-differential equations which cannot be solved in closed form. One usually relies on simulations, for example, by test-particle methods in the BUU approach. In particular, the collision term is evaluated stochastically. In such simulations, some of the implementations are not necessarily dictated by the underlying equations and are handled differently in the numerical codes. Hence-even within the same theoretical framework-different versions of codes with varying procedures and inputs have been developed, though are often not 
properly documented. Thus, seemingly similar calculations have led to substantially different results. Clearly, this is an unsatisfactory state of affairs. This situation has stimulated various efforts for code comparison [133-136]. Earlier attempts to analyze the situation took place in workshops at Trento in 2004 (for particle production at $1 \mathrm{GeVu}^{-1}$ [133]) and in 2009 (for flow at $100 \mathrm{MeVu}^{-1}$ and $400 \mathrm{MeVu}^{-1}$ ). During the 2009 workshop calculations of the main transport codes with identical physical inputs were compared with respect to the main global observables - such as yields, rapidity distributions and flow observables. Although in general good qualitative agreement was found, the degree of quantitative agreement requires improved understanding of the calculated observables in order to test finer details of the EOS, particularly those related to the symmetry energy. To clarify the source of these discrepancies, internal quantities, such as the number, energy distribution, and blocking of collisions, were investigated and, indeed, showed large differences. This is significant, since differences in the collision term can strongly influence particle production near thresholds.

A consensus among the practitioners of the transport codes is that a continuation and stabilization of such comparisons is a worthy and timely project. Indeed, a new workshop in China where many groups are engaged in transport calculations using a variety of codes has been organized. The aim of the workshop is to compare, verify, and validate the reliability of the most widely used codes, and to document properly versions of the codes together with benchmark examples. This endeavor should strengthen considerably the impact of heavy-ion research on the investigation of the nuclear EOS - particularly on the symmetry energy at high densities. From the remaining differences between codes it will allow to estimate systematic theoretical uncertainities.

\section{Conclusions and a way forward}

The last few years have seen enormous progress in our understanding of the density dependence of the symmetry energy. However, significant challenges lie ahead. The present document provides a roadmap that continues to foster dialogue and promotes collaborations between the astrophysics and the nuclear-physics communities. We articulate a way forward in areas of relevance to the symmetry energy - such as nuclear structure, heavy-ion collisions, and neutron stars-by proposing new terrestrial experiments and astrophysical observations at next generation facilities. In what follows we summarize our vision for the future of the symmetry energy.

\subsection{A way forward at very low densities}

The symmetry energy at very low densities is of great relevance to the neutrinosphere region in core collapse supernovae. The neutrinosphere-the surface of last scattering for the neutrinos-is composed of a low density gas of neutron-rich matter at temperatures of about $T=5 \mathrm{MeV}$ and densities of $\rho \approx 0.01 \rho_{0}$. Under these conditions neutron-rich matter is unstable against cluster formation, as the formation of light nuclei is energetically favorable. The existence of light clusters could modify the neutrino opacity and ultimately affect the conditions for nucleosynthesis. Remarkably, many properties of the neutrinosphere may be simulated in the collision of heavy ions. New experiments involving heavy-ion collisions with very neutron-rich (or proton-rich) nuclei at present and near future radioactive beam facilities will be able to reproduce many neutrinosphere conditions. Moreover, measurement of the yields and distribution of light fragments may help elucidate the dynamics of the neutrinosphere. In particular, comparing these yields to transport models with improved 
descriptions of light clusters should allow more accurate supernova simulations of neutrino spectra and nucleosynthesis.

\subsection{A way forward with neutron skins}

The neutron-skin thickness of heavy nuclei is a strong isovector indicator that correlates strongly to the density dependence of the symmetry energy-particularly to the symmetry slope $L$. The sensitivity of $R_{\text {skin }}$ to $L$ emerges from a competition between surface tension and the difference between the symmetry energy at the center and surface of the nucleus. In particular, for a stiff symmetry energy, it is favorable to push the excess neutrons to the surface where the symmetry energy is small. Thus, models with large $L$ tend to produce thicker skins. The upcoming PREX-II experiment promises to determine the neutron-skin thickness of ${ }^{208} \mathrm{~Pb}$ with a $\pm 0.06 \mathrm{fm}$ accuracy. Using the strong correlation between $R_{\text {skin }}^{208}$ and $L$ leads to a determination of $L$ with an accuracy of $\Delta L_{\exp }=40.8 \mathrm{MeV}$. Given that the model predictions suggest an intrinsic theoretical error of $\Delta L_{\mathrm{th}}=6.8 \mathrm{MeV}$ (see equation (9)), we expect a cumulative error in the determination of $L$ of

$$
\Delta L=\sqrt{\Delta L_{\mathrm{exp}}^{2}+\Delta L_{\mathrm{th}}^{2}}=41.4 \mathrm{MeV} .
$$

We note that the quoted theoretical error may be underestimated, as the set of models used to extract such a value, although representative, is not unique. Can one improve on the limit on $\Delta L$ ? Clearly, if a follow-up to PREX-II is feasible and the statistical accuracy of $\pm 0.02 \mathrm{fm}$ is attained, then the error in $L$ could be significantly reduced to only $\Delta L=15.2 \mathrm{MeV}$.

Whereas PREX-II will place a stringent constraint on the density dependence of the symmetry energy (particularly on $L$ ), models predicting neutron radii of medium mass and light nuclei are affected by nuclear dynamics beyond $L$. In particular, the calcium radius experiment (CREX) will provide new and unique input into the isovector sector of nuclear theories and the high precision measurement of $R_{n}^{48}( \pm 0.02 \mathrm{fm})$ will help build a critical bridge between $a b$ initio approaches [137] and nuclear density functional theory [138]. Note that CREX results can be directly compared to new coupled cluster calculations that are sensitive to three-neutron forces [139, 140]. Moreover, both ${ }^{48} \mathrm{Ca}$ and ${ }^{208} \mathrm{~Pb}$ - which are the basis of the approved CREX and PREX-II experiments [141] — are doubly-magic with a relatively simple nuclear structure, making them ideal candidates for accurate measurement of the parity-violating asymmetry. In particular, the closed-shell nature of both nuclei results in a large energy gap (of a few MeV) to the first excited state. In combination with the high-resolution spectrometers at Jefferson Lab, this has the enormous advantage of ensuring that only the elastic electrons are captured in the detectors.

Finally, other nuclei such as ${ }^{120} \mathrm{Sn}$ and its heavier stable isotopes as well as measurements at other momentum transfers needed to map out the nuclear surface, should be considered. However, it appears that measurements on open-shell nuclei such as Sn may not be as feasible or as cleanly interpretable as PREX and CREX [142]. At present it is unclear whether any of the stable Sn-isotopes may display a higher sensitivity to $L$ than ${ }^{48} \mathrm{Ca}$ or ${ }^{208} \mathrm{~Pb}$. Thus, an important next step is to generate 'sensitivity plots' for several semi-magic nuclei. Ultimately, the concordance between CREX, PREX, and TREX ('Tin Radius EXperiment') may provide the best hope for a stringent constraint on the density dependence of the symmetry energy.

\subsection{A way forward with giant resonances}

Besides the neutron-skin of heavy nuclei, the distribution of isoscalar monopole strength has been found to be sensitive to the density dependence of the symmetry energy. Unfortunately, 
this sensitivity is hindered by the relatively small neutron excess of the stable nuclei explored to date. Thus, an important step forward is to measure the distribution of monopole strength in exotic (very neutron-rich) nuclei at next generation radioactive beam facilities. In particular, mapping GMR centroid energies outside the stable ${ }^{112} \mathrm{Sn}-{ }^{124} \mathrm{Sn}$ region is likely to provide valuable insights. Moreover, such an experimental campaign may also help elucidate the softness of Tin.

In the case of the isovector giant dipole resonance (GDR), the electric dipole polarizability $\alpha_{\mathrm{D}}$ was shown to be a strong isovector indicator. In particular, the combination of $\alpha_{\mathrm{D}}^{208}$ times the symmetry energy at saturation density $J$ was seen to be strongly correlated to the neutron-skin thickness $R_{\text {skin }}^{208}$. Although photo-absorption experiments have been used for decades to probe the structure of the GDR, it is critical to delve into the low-energy region for a proper account of $\alpha_{\mathrm{D}}$. Indeed, for the case of the exotic ${ }^{68} \mathrm{Ni}$ nucleus, the PDR alone accounts for about $25 \%$ of $\alpha_{\mathrm{D}}$. Here too the systematic exploration of the isovector dipole strength along the chain of Sn-isotopes, for both stable and exotic nuclei, may provide new insights into the problem.

\subsection{A way forward in theory}

An accurately calibrated microscopic theory that both predicts and provides well-quantified theoretical uncertainties is one of the goals of modern nuclear energy density functionals (EDFs) [101, 143]. The need to quantify model uncertainties in an area such as theoretical nuclear physics is particularly urgent as models that are fitted to experimental data are then used to extrapolate to the extremes of density and isospin asymmetry. Ambitious theoretical programs aimed at calibrating future EDFs using ground-state properties of finite nuclei, their collective response, and neutron-star properties-supplemented by a proper covariance analysis - are well on their way. A promising first step along these lines has already been taken in [144].

\subsection{A way forward in astrophysics}

There is now a small number of neutron stars with joint mass and radius constraints from spectral fitting. Constraining the EOS by a joint fit is a powerful means of extracting information about dense matter. To realize this potential will require the elimination of systematic errors in distance and spectral fitting. With the anticipated parallax measurements from the Gaia mission, the uncertainty in globular cluster distances will be drastically reduced. Furthermore, the distances to nearby field neutron stars with identified companions, such as Cen X-4, will be known. Nearby neutron stars, with a higher flux, would make a good target for spectral fitting. Spectroscopy of the companion might also constrain the composition of the accreted material and therefore the emergent spectrum. Improved distances would also help in the determination of mass and radius from x-ray bursts, if reliable models of the spectral evolution during the burst can be made. Future timing missions such as $L O F T^{10}$ and $N I C E R^{11}$ may provide further constraints on the mass-radius relation via rotation-resolved spectroscopy of pulsations. It may also be possible to extract the compactness $M / R$ from observations of spectral edges observed in bursts with strong photospheric expansion [145, 146].

Further work should explore the implications of a small $(9 \mathrm{~km})$ neutron star radius. Such a small radius implies a softening of the symmetry energy for $\rho_{0} \lesssim \rho \lesssim 2 \rho_{0}$, which can be probed by heavy-ion collisions. The softening needed for such small radii also suggests

${ }^{10}$ Large Observatory For x-ray Timing www.isdc.unige.ch/loft/.

${ }^{11}$ Neutron star Interior Composition ExploreR http://heasarc.gsfc.nasa.gov/docs/nicer/. 
that the proton fraction remains low, and might preclude the onset of direct Urca neutrino cooling. Further searches for cold, isolated neutron stars, as well as faint qLMXBs such as SAX J1808.4-3658 and 1H $1905+00$ can inform us about the distribution of neutron stars with enhanced neutrino cooling.

Finally, the symmetry energy influences other quantities, such as the crust/core transition density and the fractional moment of inertia of the crust; it also affects the region of the crust with strongly deformed nuclei, the 'pasta' phase. These affect transport properties, such as the Ohmic resistivity of the neutron star crust, that might play a role in other observable phenomena (see, e.g., [147]).

\subsection{A way forward for heavy-ion collisions}

Heavy-ion collisions provide the only means to study the density, isospin, and temperature dependence of the equation of state (EOS) under controlled laboratory conditions. The large dynamic range accessible with heavy-ion collisions allows for the exploration of the symmetry energy in very dilute matter up to several times the saturation density. Specifically, the region at about twice saturation density is critical for the determination of neutron-star radii. Placing strong constraints on the high-density component of the EOS of symmetric nuclear matter represents an important achievement in studies of energetic heavy-ion collision. The community is now investigating the density dependence of the symmetry energy by comparing neutron and proton observables, and in general isospin partners in light clusters, using reactions with different neutron-proton asymmetries. Already a series of experiments of heavy-ion collisions with energies in the Fermi energy range have constrained the symmetry energy from about $20 \%$ above $\rho_{0}$ down to about $1 / 3 \rho_{0}$. These constraints agree well with, and in some cases even sharpen, the constraints from nuclear structure studies of exotic nuclei. With the availability of facilities for the production of very asymmetric beams these possibilities are greatly enhanced. The present focus is to improve experimental and theoretical uncertainties of the constraints at low density and on constraining the symmetry energy at supra-saturation densities, where it is rather poorly known from microscopic calculations, and where there are very few experimental data. A way forward will be to do new experiments that take advantage of studying reactions of wide asymmetries using rare isotope beams to access a range of densities from below to well above saturation density with carefully chosen systems and observables which enhance the sensitivity to the symmetry energy.

Transport calculations are essential to extract the information about the equation of state from the complex heavy-ion collision process. By considering appropriate observables, like ratios of isospin-sensitive quantities, it has already been possible to obtain much information about the symmetry energy. But it has also been learned that transport calculations have to be refined to give a better description of fluctuations, cluster and particle production, and generally have to improve the consistency and reproducibility between different codes. A planned series of workshops on code evaluation and comparison is an important step in this direction.

\subsection{A way forward for dense $Q C D$}

Quantum chromodynamics is presently the ultimate theory of the strong interaction. As such, it determines the rich and complex structure of the phase diagram of strongly interacting matter. At low baryon density and high temperatures, collisions of heavy ions at facilities of increasing energy and sophistication are providing hints on the transition from hadronic matter to deconfined quark matter. At the opposite extreme, namely, high densities and low 
temperatures, neutron stars offer the best (and perhaps unique) view of cold, dense matter. The Facility for Antiproton and Ion Research (FAIR) in Europe provides the best alternative at bridging the gap. However, theoretical guidance into the detailed structure of the QCD phase diagram is hindered by the intrinsically non-perturbative nature of QCD. Although much progress has been made, enormous challenges remain. For example, QCD predicts that at ultra-high densities and low temperatures - where the up, down, and strange quarks are effectively massless - the ground state is a superconductor with a unique 'color-flavor locking' pairing scheme [148]. Unfortunately, the enormous densities that may be required for this phase to develop may not be reached in the stellar cores. So the QCD ground state at the densities of relevance to neutron stars remains an important challenge. On the other hand, lattice QCD provides powerful insights into the nature of the phase (or rather crossover) transition at finite temperature and zero baryon density (or equivalently zero chemical potential $\mu$ ). While at present lattice simulations at arbitrary values of the chemical potential are hindered by the vexing 'sign problem', renewed interest in the QCD phase diagram has seen the emergence of various alternatives, including a Taylor series (akin to the virial expansion) in the small parameter $\mu / T$. In particular, the main virtue of these exact lattice results is that they provide critical benchmarks for assessing the reliability of other theoretical approaches.

\section{Acknowledgments}

This work reports results from the first International Collaborations in Nuclear Theory (ICNT) program at NSCL/FRIB during 2013. We acknowledge the generous financial and logistic support from the director of NSCL/FRIB, which made the ICNT program possible. The program was co-hosted by the grants-in-aid for Scientific Research on Innovative Areas (nos. 24105001, 24105008, area no. 2404) from JSPS. CJH acknowledges support from DOE grants DE-FG02-87ER40365 (Indiana University) and DE-SC0008808 (NUCLEI SciDAC Collaboration). EFB acknowledges support from NSF AST grant 11-09176. YK acknowledges support from the Rare Isotope Science Project funded by the Ministry of Science, ICT and Future Planning (MSIP) and National Research Foundation (NRF) of KOREA. MBT and WGL acknowledge the funding of NSF under grant no. PHY-0606007 and DOE grant no. DE-SC0004835. JP acknowledges support from DOE grant no. DE-FD05-92ER40750. HHW acknowledges support from the Excellence Cluster 'Origin and Structure of the Universe' of the German Research Foundation (DFG).

\section{References}

[1] von Weizsacker C F 1935 Z. Phys. 96431

[2] Bethe H A and Bacher R F 1936 Rev. Mod. Phys. 882

[3] Piekarewicz J and Centelles M 2009 Phys. Rev. C 79054311

[4] Baym G, Pethick C and Sutherland P 1971 Astrophys. J. 170299

[5] Ruester S B, Hempel M and Schaffner-Bielich J 2006 Phys. Rev. C 73035804

[6] Roca-Maza X and Piekarewicz J 2008 Phys. Rev. C 78025807

[7] Steiner A W, Prakash M, Lattimer J M and Ellis P J 2005 Phys. Rep. 411325

[8] Tsang M B et al 2012 Phys. Rev. C 86015803

[9] Yakovlev D G and Pethick C J 2004 Ann. Rev. Astron. Astrophys. 42169

[10] Horowitz C J and Schwenk A 2006 Nucl. Phys. A 77655

[11] Natowitz J B et al 2010 Phys. Rev. Lett. 104202501

[12] Borsányi S, Fodor Z, Katz S D, Krieg S, Ratti C and Szabó K 2012 J. High Energy Phys. JHEP01(2012)138

[13] Bazavov A et al 2012 Phys. Rev. D 86034509 
[14] Roth R, Binder S, Vobig K, Calci A, Langhammer J and Navrátil P 2012 Phys. Rev. Lett. 109052501

[15] Krüger T, Tews I, Hebeler K and Schwenk A 2013 Phys. Rev. C 88025802

[16] Gandolfi S, Carlson J, Reddy S, Steiner A W and Wiringa R B 2013 arXiv:1307.5815

[17] Katayama T and Saito K 2013 Phys. Rev. C 88035805

[18] Sammarruca F 2011 Phys. Rev. C 84044307

[19] Lee H K, Park B-Y and Rho M 2011 Phys. Rev. C 83025206

[20] Dong H, Kuo T, Lee H K, Machleidt R and Rho M 2012 arXiv:1207.0429

[21] Kim Y, Seo Y, Shin I J and Sin S-J 2011 J. High Energy Phys. JHEP06(2011)011

[22] Arcones A, Martínez-Pinedo G, O'Connor E, Schwenk A, Janka H-T, Horowitz C J and Langanke K 2008 Phys. Rev. C 78015806

[23] Roberts L F 2012 Astrophys. J. 755126

[24] Roberts L F, Reddy S and Shen G 2012 Phys. Rev. C 86065803

[25] Martínez-Pinedo G, Fischer T, Lohs A and Huther L 2012 Phys. Rev. Lett. 109251104

[26] Hofstadter R 1956 Rev. Mod. Phys. 28214

[27] Fricke G, Bernhardt C, Heilig K, Schaller L A, Schellenberg L, Shera E B and de Jager C W 1995 At. Data Nucl. Data Tables 60177

[28] Angeli I and Marinova K 2013 At. Data Nucl. Data Tables 9969

[29] Ray L and Hoffmann G W 1985 Phys. Rev. C 31538

[30] Ray L, Hoffmann G W and Coker W R 1992 Phys. Rep. 212223

[31] Piekarewicz J and Weppner S 2006 Nucl. Phys. A 77810

[32] Abrahamyan S et al 2012 Phys. Rev. Lett. 108112502

[33] Horowitz C et al 2012 Phys. Rev. C 85032501

[34] Aniol K A et al 1999 Phys. Rev. Lett. 821096

[35] Aniol K A et al 2004 Phys. Rev. C 69065501

[36] Aniol K A et al 2006 Phys. Rev. Lett. 96022003

[37] Acha A et al 2007 Phys. Rev. Lett. 98032301

[38] Ahmed Z et al 2012 Phys. Rev. Lett. 108102001

[39] Beck D H 1989 Phys. Rev. D 393248

[40] Armstrong D S et al 2005 Phys. Rev. Lett. 95092001

[41] Androić D et al 2010 Phys. Rev. Lett. 104012001

[42] Androic D et al (Qweak Collaboration) 2013 Phys. Rev. Lett. 111141803

[43] Donnelly T W, Dubach J and Sick I 1989 Nucl. Phys. A 503589

[44] Horowitz C J, Pollock S J, Souder P A and Michaels R 2001 Phys. Rev. C 63025501

[45] Roca-Maza X, Centelles M, Vinas X and Warda M 2011 Phys. Rev. Lett. 106252501

[46] Reinhard P G et al 2013 Phys. Rev. C 88034325

[47] Centelles M, Roca-Maza X, Viñas X and Warda M 2009 Phys. Rev. Lett. 102122502

[48] Farine M, Pearson J and Rouben B 1978 Nucl. Phys. A 304317

[49] Brown B A 2013 arXiv:1308.3664

[50] Zhang Z and Chen L-W 2013 arXiv:1302.5327

[51] Li T et al 2007 Phys. Rev. Lett. 99162503

[52] Li T et al 2010 Phys. Rev. C 81034309

[53] Piekarewicz J 2007 Phys. Rev. C 76031301

[54] Reinhard P-G and Nazarewicz W 2010 Phys. Rev. C 81051303

[55] Piekarewicz J et al 2012 Phys. Rev. C 85041302

[56] Myers W D and Swiatecki W J 1974 Ann. Phys. 84186

[57] Stringari S and Lipparini E 1982 Phys. Lett. B 117141

[58] Satula W, Wyss R A and Rafalski M 2006 Phys. Rev. C 74011301

[59] Roca-Maza X et al 2013 Phys. Rev. C 88024316

[60] Tamii A et al 2011 Phys. Rev. Lett. 107062502

[61] Poltoratska I et al 2012 arXiv:1203.2155

[62] Fattoyev F and Piekarewicz J 2013 Phys. Rev. Lett. 111162501

[63] Col G, Garg U and Sagawa H 2013 arXiv:1309.1572

[64] Lindblom L 1992 Astrophys. J. 398569

[65] Demorest P B, Pennucci T, Ransom S M, Roberts M S E and Hessels J W T 2010 Nature 4671081

[66] Antoniadis J et al 2013 Science 340448

[67] Özel F, Güver T and Psaltis D 2009 Astrophys. J. 6931775

[68] Güver T, Wroblewski P, Camarota L and Özel F 2010 Astrophys. J. 7191807 
[69] Steiner A W, Lattimer J M and Brown E F 2010 Astrophys. J. 72233

[70] Suleimanov V, Poutanen J, Revnivtsev M and Werner K 2011 Astrophys. J. 742122

[71] Güver T, Psaltis D and Özel F 2012 Astrophys. J. 74776

[72] Güver T, Özel F and Psaltis D 2012 Astrophys. J. 74777

[73] Zamfir M, Cumming A and Galloway D K 2012 Astrophys. J. 74969

[74] Bildsten L, Salpeter E E and Wasserman I 1992 Astrophys. J. 384143

[75] Steiner A W, Lattimer J M and Brown E F 2013 Astrophys. J. Lett. 765 L5

[76] Guillot S, Servillat M, Webb N A and Rutledge R E 2013 Astrophys. J. 7727

[77] Lattimer J M and Prakash M 2007 Phys. Rep. 442109

[78] Lattimer J M and Steiner A W 2013 arXiv:1305.3242

[79] Heyl J S et al 2012 Astrophys. J. 76151

[80] Pancino E, Bellazzini M and Marinoni S 2013 Mem. Soc. Astron. Italiana 8483

[81] Lattimer J M and Prakash M 2001 Astrophys. J. 550426

[82] Tsuruta S and Cameron A G W 1965 Nature 207364

[83] Lattimer J M, Pethick C J, Prakash M and Haensel P 1991 Phys. Rev. Lett. 662701

[84] Page D, Lattimer J M, Prakash M and Steiner A W 2009 Astrophys. J. 7071131

[85] Kaplan D L, Frail D A, Gaensler B M, Gotthelf E V, Kulkarni S R, Slane P O and Nechita A 2004 Astrophys. J. Suppl. 153269

[86] Kaplan D L, Gaensler B M, Kulkarni S R and Slane P O 2006 Astrophys. J. Suppl. 163344

[87] Sato K 1979 Prog. Theor. Phys. 62957

[88] Haensel P and Zdunik J L 1990 Astron. Astrophys. 227431

[89] Steiner A W 2012 Phys. Rev. C 85055804

[90] Brown E F, Bildsten L and Rutledge R E 1998 Astrophys. J. Lett. 504 L95

[91] Yakovlev D G, Levenfish K P, Potekhin A Y, Gnedin O Y and Chabrier G 2004 Astron. Astrophys. 417169

[92] Heinke C O, Jonker P G, Wijnands R and Taam R E 2007 Astrophys. J. 6601424

[93] Brown E F, Bildsten L and Chang P 2002 Astrophys. J. 574920

[94] Jonker P G, Steeghs D, Chakrabarty D and Juett A M 2007 Astrophys. J. Lett. 665 L147

[95] Danielewicz P, Lacey R and Lynch W G 2002 Science 2981592

[96] Fuchs C and Wolter H H 2006 Eur. Phys. J. A 305

[97] Tsang M B, Zhang Y, Danielewicz P, Famiano M, Li Z, Lynch W G and Steiner A W 2009 Phys. Rev. Lett. 102122701

[98] Kohley Z et al 2013 Phys. Rev. C 88041601

[99] Danielewicz P and Lee J 2013 arXiv:1307.4130

[100] Tamii A, von Neumann-Cosel P and Poltoratska I Eur. Phys. J. A at press arXiv:1307.2706

[101] Kortelainen M et al 2010 Phys. Rev. C 82024313

[102] Baran V, Colonna M, Di Toro M, Greco V, Zielinska-Pfabé M and Wolter H H 2002 Nucl. Phys. A 703603

[103] Ono A, Danielewicz P, Friedman W A, Lynch W G and Tsang M B 2003 Phys. Rev. C 68051601

[104] Typel S, Röpke G, Klähn T, Blaschke D and Wolter H H 2010 Phys. Rev. C 81015803

[105] Coupland D D S, Lynch W G, Tsang M B, Danielewicz P and Zhang Y 2011 Phys. Rev. C 84054603

[106] Zhang Y, Li Z, Zhou C and Tsang M B 2012 Phys. Rev. C 85051602

[107] Amorini F et al 2009 Phys. Rev. Lett. 102112701

[108] Cardella G et al 2012 Phys. Rev. C 85064609

[109] Rizzo J, Colonna M, Baran V, Toro M D, Wolter H and Zielinska-Pfabe M 2008 Nucl. Phys. A 80679

[110] De Filippo E et al 2012 Phys. Rev. C 86014610

[111] Alford M G, Rajagopal K and Wilczek F 1999 Nucl. Phys. B 537443

[112] Brown B A 2000 Phys. Rev. Lett. 855296

[113] Xu C and Li B-A 2010 Phys. Rev. C 81064612

[114] Ferini G, Gaitanos T, Colonna M, di Toro M and Wolter H H 2006 Phys. Rev. Lett. 97202301

[115] Reisdorf W et al (Fopi Collaboration) 2007 Nucl. Phys. A 781459

[116] Reisdorf W et al (Fopi Collaboration) 2012 Nucl. Phys. A 8761

[117] Giordano V, Colonna M, di Toro M, Greco V and Rizzo J 2010 Phys. Rev. C 81044611

[118] Russotto P et al 2011 Phys. Lett. B 697471

[119] Cozma M D, Leifels Y, Trautmann W, Li Q and Russotto P 2013 Phys. Rev. C 88044912

[120] Ferini G, Colonna M, Gaitanos T and di Toro M 2005 Nucl. Phys. A 762147 
[121] Xiao Z, Li B-A, Chen L-W, Yong G-C and Zhang M 2009 Phys. Rev. Lett. 102062502

[122] Feng Z-Q and Jin G-M 2010 Phys. Lett. B 683140

[123] Hong J and Danielewicz P 2013 arXiv:1307.7654

[124] Prassa V, Gaitanos T, Ferini G, di Toro M, Lalazissis G A and Wolter H H 2010 Nucl. Phys. A 83288

[125] Lopez X et al 2007 Phys. Rev. C 75011901

[126] Colonna M 2013 Phys. Rev. Lett. 110042701

[127] Ono A 2013 J. Phys.: Conf. Ser. 420012103

[128] Ono A 2013 J. Phys.: Conf. Ser. 436012068

[129] Li B-A and Chen L-W 2005 Phys. Rev. C 72064611

[130] Furuta T and Ono A 2006 Phys. Rev. C 74014612

[131] Baran V, Colonna M, di Toro M and Larionov A B 1998 Nucl. Phys. A 632287

[132] Papa M 2013 Phys. Rev. C 87014001

[133] Kolomeitsev E E et al 2005 J. Phys. G: Nucl. Part. Phys. 31741

[134] Kohley Z et al 2012 Phys. Rev. C 85064605

[135] Colonna M, Ono A and Rizzo J 2010 Phys. Rev. C 82054613

[136] 2014 Int. Workshop on Simulations of Low and Intermediate Energy Heavy Ion Collisions (Shanghai, China, 8-12 January 2014) www.physics.sjtu.edu.cn/hic2014/

[137] Forssén C, Hagen G, Hjorth-Jensen M, Nazarewicz W and Rotureau J 2013 Phys. Scr. T 152 014022

[138] Bender M, Heenen P-H and Reinhard P-G 2003 Rev. Mod. Phys. 75121

[139] Hagen G, Hjorth-Jensen M, Jansen G R, Machleidt R and Papenbrock T 2012 Phys. Rev. Lett. 109032502

[140] 2013 Calcium Radius Experiment (CREX) Workshop at Jefferson Lab (Newport News, VA, 17-19 March 2013) www.jlab.org/conferences/crex/

[141] 2014 The PREX and CREX Proposals http://hallaweb.jlab.org/parity/prex

[142] Ban S, Horowitz C J and Michaels R 2012 J. Phys. G: Nucl. Part. Phys. 39015104

[143] UNEDF Collaboration 2012 Building a Universal Nuclear Energy Density Functional http://unedf.org

[144] Erler J, Horowitz C, Nazarewicz W, Rafalski M and Reinhard P-G 2012 arXiv:1211.6292

[145] Weinberg N N, Bildsten L and Schatz H 2005 Astrophys. J. 6391018

[146] 't Zand J J M and Weinberg N N 2010 Astron. Astrophys. 520 A81

[147] Pons J A, Vigano D and Rea N 2013 Nature Phys. 9431

[148] Alford M G, Schmitt A, Rajagopal K and Schäfer T 2008 Rev. Mod. Phys. 801455 